\title{
Data-Driven Epidemic Intelligence Strategies Based on Digital Proximity Tracing Technologies in the Fight against COVID-19 in Cities
}

\author{
Dario Esposito $^{1, *(\mathbb{D}}$, Giovanni Dipierro ${ }^{2}\left(\mathbb{D}\right.$, Alberico Sonnessa ${ }^{1}\left(\mathbb{D}\right.$, Stefania Santoro $^{1}\left(\mathbb{D}\right.$, Simona Pascazio $^{3}$ \\ and Irene Pluchinotta ${ }^{4}$ (i)
}

1 Department of Civil, Environmental, Land, Construction and Chemistry (DICATECh), Polytechnic University of Bari, 70125 Bari, Italy; alberico.sonnessa@poliba.it (A.S.); stefania.santoro@poliba.it (S.S.)

2 Independent expert-consultant; giovanni.dipierro1@gmail.com

3 Eunomia Studio di Avvocati, 70121 Bari, Italy; s.pascazio@eunomiastudio.it

4 Institute for Environmental Design and Engineering, The Bartlett Faculty of the Built Environment, University College London, London WC1H 0NN, UK; i.pluchinotta@ucl.ac.uk

* Correspondence: dario.esposito@poliba.it

check for updates

Citation: Esposito, D.; Dipierro, G.; Sonnessa, A.; Santoro, S.; Pascazio, S.; Pluchinotta, I. Data-Driven Epidemic Intelligence Strategies Based on Digital Proximity Tracing Technologies in the Fight against COVID-19 in Cities. Sustainability 2021, 13, 644. https://doi.org/ $10.3390 /$ su13020644

Received: 9 November 2020

Accepted: 5 January 2021

Published: 12 January 2021

Publisher's Note: MDPI stays neutral with regard to jurisdictional clai$\mathrm{ms}$ in published maps and institutional affiliations.

Copyright: (C) 2021 by the authors. Licensee MDPI, Basel, Switzerland. This article is an open access article distributed under the terms and conditions of the Creative Commons Attribution (CC BY) license (https:// creativecommons.org/licenses/by/ $4.0 /)$.

\begin{abstract}
In a modern pandemic outbreak, where collective threats require global strategies and local operational defence applications, data-driven solutions for infection tracing and forecasting epidemic trends are crucial to achieve sustainable and socially resilient cities. Indeed, the need for monitoring, containing, and mitigating the ongoing COVID-19 pandemic has generated a great deal of interest in Digital Proximity Tracing Technology (DPTT) on smartphones, as well as their function and effectiveness and insights of population acceptance. This paper introduces and compares different Data-Driven Epidemic Intelligence Strategies (DDEIS) developed on DPTTs. It aims to clarify to what extent DDEIS could be effective and both technologically and socially suitable in reaching the objective of a swift return to normality for cities, guaranteeing public health safety and minimizing the risk of epidemic resurgence. It assesses key advantages and limits in supporting both individual decision-making and policy-making, considering the role of human behaviour. Specifically, an online survey carried out in Italy revealed user preferences for DPTTs and provided preliminary data for an SEIR (Susceptible-Exposed-Infectious-Recovered) epidemiological model. This was developed to evaluate the impact of DDEIS on COVID-19 spread dynamics, and results are presented together with an evaluation of potential drawbacks.
\end{abstract}

Keywords: future healthy cities; epidemic control; infection tracing; SARS-CoV-2; digital contact tracing; model-driven strategies; SEIR; big data-driven policy making

\section{Introduction}

During the period from 2011 to 2018, the World Health Organization (WHO) recorded 1483 epidemic events in 172 countries [1]. Epidemic-prone diseases can lead to severe consequences on population health and economy of the affected countries, depending on several factors (e.g., strength of the pathogen, sanitary conditions, food quality, pollution levels, healthcare system efficiency) and have a global impact when they turn into a pandemic [2]. The threat of a global health crisis emerged at the end of December 2019, when the WHO was informed by the Chinese government of anomalous pneumonia cases in Wuhan, originating from an unknown virus, today well-known as Severe Acute Respiratory Syndrome-Coronavirus-2 (SARS-CoV-2) ("World Health Organization" n.d.). From January 2020, the virus has spread beyond the Chinese borders, causing a pandemic. To date (28 October 2020), about 44 million people have been infected, and 1,200,000 people have died worldwide [3]. In addition, the pandemic caused by the SARS-CoV-2 virus, officially named Coronavirus Disease-2019 (COVID-19), is having a devastating impact on global trade, economy, and social stability worldwide, representing one of the worst 
health, economic, and social crises faced by the world population after World War II [4,5]. Furthermore, even the countries where the spread of the infection seemed to be under control (e.g., in Europe) are now facing the second wave related to the recurrence of the virus [3]. Until a vaccine is available, a variety of measures and strategies have been put in place to contain this threat. Immediately after the outbreak of COVID-19, quarantine and total lockdown proved to be successful in reducing the virus transmission [6]. However, along with the use of physical distancing, sanitization, and restrictions of public gatherings, the swift response to the spread of the infection requires accurate data and information collection to support the implementation of control measures.

In today's world, characterized by widespread digitalization, data-driven technologies supported by close-range localization can provide rapid and consistent data, which can play a key role in developing strategies in protecting the population and assisting in the fight against COVID-19. Data-Driven Epidemic Intelligence Strategies (DDEIS) based on Digital Proximity Tracing Technologies (DPTT) might represent a proactive approach to tackle the COVID-19 pandemic, and their combined use could work as a third-millennium digital vaccine to attain herd protection [7]. These could be used as an efficient and cost-effective measure to control the spread, preventing the virus from propagating exponentially within the population, avoiding mass interventions such as lockdown [8] and helping to minimize the proportion of uninfected people forced into quarantine, limiting restrictions to those most at risk [9]. On the other hand, the adopted DPTTs suffer from a degree of privacy invasion, whose acceptance depends on the legal and political context where they originated. For example, the Chinese government adopted an approach based on the strengthening of an existing surveillance system that is highly invasive in terms of personal privacy. By contrast, in European countries where data protection rights are well-established, less invasive technologies were adopted. However, effectiveness of DDEIS based on DPTT coexisting with individual freedom in slowing down the spread has yet to be reached [10]. Furthermore, it is not only privacy concerns that DPTT needs to face. An assumption is that such approaches might not have much impact where an extensive community transmission has already occurred [11]. Other issues range from technical limitations, e.g., accuracy, sensitivity, and data granularity, to the absence of evidence that DPTT effectively fights the pandemic, due barriers to deployment and security and social risks [12,13]. Finally, although some attempts to define a taxonomy of typology of public health solutions relying on digital technologies were made [14], these were limited on a wider scale, where a more fine-grained distinction of DDEIS on DPTT detail analysis and an evaluation of implications and perspectives for decision-making are still missing. This regrettably results in overlapping descriptions and confusion of usability. This uncertainty in turn hinders their applicability over the entire range and causes their rejection by public opinion.

Within this context, this paper aims to define and compare from different perspectives DDEIS based on DPTT in order to clarify the context in which these strategies could be chosen and implemented by national governments and local decision-makers. Specifically, their functions and purposes are discussed, and population response is evaluated with a preliminary online survey (conducted in Italy). Furthermore, DDEIS impacts are assessed through a modified SEIR (Susceptible-Exposed-Infectious-Recovered) epidemiological model in order to identify and evaluate the advantages and limitations of their implementation in the subsequent phases of a pandemic and for a return to a normal life.

The paper is structured as follows: after the present introduction, Section 2 describes the background of the epidemic spread and the conventional measures adopted. In Section 3, four DDEIS are presented together with their functioning and scope. Section 4 reports the results of the survey that are relevant to the present study. Section 5 provides the SEIR epidemiological model used to assess the effectiveness of the proposed DDEIS. Results and discussions follow in Section 6, and concluding remarks are presented in the final section. 


\section{Background}

As the pandemic spread, health organizations implemented several countermeasures to bring it under control. The spotlight was on the basic reproduction number R0, defined as the number of secondary infections produced by one primary infection in a fully susceptible population. At population level, if $\mathrm{R} 0$ is higher than 1, the epidemic rises, while if R0 is less than 1, it is destined to peter out. Restrictive measures, such as quarantine, lower the R0 index. However, R0 can also increase, with the risk of a complete resurgence, when restrictions are relaxed or lifted [15]. The conventional strategy aimed at bringing and maintaining R0 under the critical threshold involves a combination of measures, also known as test, trace, and treat. The manual contact tracing process, conducted by public health officials, consists of tracking down anyone who may have been exposed to SARSCoV-2 due to a contact with an infected person (also during the incubation period), in order to alert potential new cases to seek testing and monitoring for symptoms. This protocol is traditionally deployed manually by interviewing infected subjects and assisting them in recalling past movements and interactions. This procedure is resource-demanding, time-consuming, and proportionally too labour-intensive, while outputs have proved to be inaccurate and limited [16]. Besides this, population screening for SARS-CoV-2 is essential in suppressing its spread. However, a large capacity for community testing requires the availability of personnel, infrastructures, and equipment. The ECDC reported that in the EU, only people suffering symptoms acute enough for hospitalization were tested [17]. Nevertheless, symptom-based control alone, i.e., testing a small proportion of highly symptomatic individuals (initially due to the scarcity of COVID-19 testing), implies a vast underestimate of infection rates per capita and thus fails to control transmission [10]. This is also caused by uncertainty about mild or asymptomatic COVID-19 carriers or recovered patients, as well as the number of self-isolating people [18]. For the same reason, it is not possible to effectively forecast where COVID-19 will spread and when the number of cases will start to fall. Indeed, studies suggest that even the total isolation of all symptomatic individuals would be insufficient to stop the epidemic since the infectiousness starts before the onset of symptoms, and the absence of symptoms does not imply absence of virus excretion $[19,20]$. Other studies indicate that almost half of COVID-19 infections are transmitted by contacts in the pre-symptomatic or asymptomatic stage [21]. This might be the reason why COVID-19 has spread easily and sufficiently quickly to cause sub-exponential (and in the worst cases exponential) growth [22]. Indeed, asymptomatic and presymptomatic transmission reduce the effectiveness of control measures if initiated by symptom onset [9]. Thus, the attempt to control the epidemic has required countries to enforce the preventative isolation and quarantine of trust of uninfected people, up to the point of mass lockdowns, since with the conventional strategy, asymptomatic or presymptomatic individuals cannot be distinguished from those who are uninfected. On average, overreacting has shown better results than not reacting; e.g., stringent pandemic policies are associated with lower mortality growth rates, especially in countries with a greater proportion of elderly and high-density urban populations and larger international travel flows [10]. Locking down entire cities was practically unavoidable, showing best results for the countries that adopted this earlier [23]. However, this entirely non-targeted measure cannot be perpetuated for too long or repeated too often, since, among its other drawbacks, it is economically highly damaging and socially disruptive. Finally, it should be carefully assessed whether lockdown is less intrusive than DPTT solutions, while their success in supporting mitigation strategies and curbing mortality growth was already proved in the case of South Korea, which avoided the drastic measure of a national lockdown [24]. Finally, a paradox needs to be addressed. Indeed, while it is on a global scale, an equivalent and interoperable cross-border DPTT system still seems to be some way from being adopted, despite the Google-Apple partnership. This is due to the large number of countries involved, a wide variety of privacy laws, different needs to protect civil rights over other priorities, and divergence in cultural attitudes and the interests of society. On the other hand, on a regional and local scale, governments are still not ready or 
aware of the value of leveraging a digital transition to collect and process detailed data to face uncertainty and support decisions. This implementation gap is caused by the lack of technological infrastructure and multidisciplinary experts. However, it is also due to public authorities struggling to deal with their inability to promptly draft ad hoc procedures, if not their widespread unpreparedness and latency in promoting an up-to-date digital mindset. Hence, too often, targeted preventive actions are not only blocked but are just not a feasible option compared to law-driven mass action enforcements [25].

Given this complex background, the extent to which DPTT-based strategies to control COVID-19 spread within cities can be adopted to reduce the risk of further lockdown and hasten a safe resumption of economic activities must be further investigated. This should involve complementary non-clinical and non-pharmaceutical measures applicable to the largest part of the population during the period required for vaccine research, clinical trials, and mass vaccination campaigns. Thus, in the following sections, DDEIS are illustrated, along with their acceptance by the population and potential impact on epidemic dynamic is evaluated and compared.

\section{Data-Driven Epidemic Intelligence Strategies}

In this section, four DDEIS based on a fine-grained DPTT distinction are proposed. These strategies could be combined to establish an underlying risk of infection transmission information system following the phases of epidemic development, which may recur cyclically through subsequent waves [26]. However, to allow for their multi-faceted comparison and potentially their alternative adoption, they are described independently.

\subsection{Early Detection}

The Early Detection strategy aims to prevent the sudden and exponential growth of contagion in a population by making faster and more precise conventional contact tracing procedures. DPTT are used only after an infected person has been identified, to automatically backtrack the chain of infection and find potentially infected people as rapidly as possible, including asymptomatic and presymptomatic subjects [9]. In this strategy, DPTT are used to record and reach both unnoticed known and unknown contacts. To implement this, the installation of apps designed for this purpose and/or the upgrading/enabling of some features (e.g., update the operating system, activation of the Bluetooth data transmitter) on mobile devices may be requested or imposed.

A risk-scoring algorithm estimates the degree of hazard run by each individual who was in contact with the infected person, relying on parameters such as distance and time of exposure, and consequently evaluates whether to issue warnings (e.g., alert messages) and address follow-up procedures, such as the targeting of tests or the enforcing of preventive quarantine/isolation, thus allowing the optimization of available resources. For instance, priority testing suspected super-spreaders allows for relieving from quarantine the larger clusters of possibly positive cases [9]. Moreover, by cross-checking data from DPTT with actual test results, proximity and duration parameters previously set in the algorithm can be fine-tuned [8]. Early Detection is particularly relevant in the initial stages of the spread of contagion, for containment purposes, but it also might prove to be effective for mitigation aims when the number of infected people makes the manual contact tracing unfeasible [7].

\subsection{Surveillance}

A Surveillance strategy allows authorities to check that each person in the population respects restrictions and regulation of the COVID-19 emergency plan. Following a detection of the infringement, fines or other actions may be pursued [27]. Surveillance is based on a centralised system for data collection and processing. Health and public safety authorities can monitor real-time personal information without consent to verify if the imposed restrictions are respected. The strategy can cross-reference DPTT data with available sources, such as surveillance camera, credit card recordings, energy bills, social media 
usage, and more and assign a hazard level to each person based on the processing of the collected information with artificial intelligence algorithms, along with the results of screening tests and traditional contact tracing [28]. Each level corresponds to restrictions and behavioural rules to be followed for a certain period of time. The movements and social relationships of the profiled subject are traced to verify if he breaks rules such as lockdown, quarantine of trust, or isolation. Surveillance is mainly used for geofencing, i.e., virtually drawing borders for certain areas or regions to limit the movement of individuals [29], by verifying whether they move away from predefined locations, such as their home or region. Moreover, with the implementation of QR-code-based features scanned at authority checkpoints, it is possible to forbid access to certain public places (e.g., shopping malls, subways) or sensitive places (emergency rooms). Although this strategy acts at an individual level, it is possible to mass-monitor the population by aggregating data to verify unauthorized mass movements or gatherings through the analysis of flows and concentrations of people. This strategy may be needed especially under the conditions of an epidemic spreading throughout large parts of a country.

\subsection{Protection}

The Protection strategy uses DPTT to allow each person to better manage spatial behaviour and physical distancing by providing users with a real-time map of the risk, displayed on their smartphones. This warns in advance about the calculated contagion risk (or, by contrast, the degree of safety) associated with a visit to a particular place or a contact with an approaching person. A similar dynamic infection risk map, that of Hospital Acquired Infection spread, is presented in Esposito [30]. This strategy works in a decentralized way, with data feeding the risk-scoring algorithm, which is delocalized on each device. Indeed, the provided service visualizes on a single map two integrated streams of information, aimed at managing both environmental contamination and social distancing. The former consists of a heat map of environmental contamination risk updated in real time. This visualizes data locations of the surroundings, reporting the likelihood of contagion for places and routes within a preset safety radius and can be complemented with alerts. This, taking into account the virus's resistance on surfaces and in environments, could indicate the safety time related to the natural decay of the virus before the access to certain places can be allowed [31,32]. Thus, it directs the user's spatial behaviour towards precautionary choices in favour of health safety, suggesting which places to avoid and for how long. Moreover, it allows us to identify where it is urgent to carry out decontamination procedures and can automatically alert competent authorities. The latter regards the information to support individual choices to improve social distancing against intra-human contagion, applying the heat map to people within a certain range from the user. This feature utilizes a contagion risk score profile of each user, based on their medical status, movements, meetings, testing results, and more, automatically and anonymously shared peer to peer with other devices within meters to estimate their users' respective infection risks and allow each smartphone algorithm to update their own realtime-computed risk [33]. The system may suggest activities based on the users' risk level, from quarantine, for lowering the gauged risk, to the need to contact health authorities. Finally, this could also implement gamification mechanisms, obtaining badges based on the behaviour of the user, e.g., respected quarantine, or on the negative result of a test. With such an app, each person would be aware at the moment of social interaction of what calculated risk they have decided to take and by contrast can feel safe to socialize freely.

Ultimately, this strategy aims to transform a non-targeted measure to a targeted one. This leads to improved social distancing measures, which often are not sufficient especially in indoor spaces where a lot of time is spent and where major infection spread occurs [34], and guides users to behave in a more conscious and safe way, informing about people or places that carry a higher risk of infection to keep the right distances and minimize the time of exposure and, if necessary, to drive users to avoid them. This strategy is useful in a de-escalation and transition phase post-lockdown and pre-vaccine to prevent epidemic 
resurgence and takes advantage of the quarantine that has been carried out, e.g., during the lockdown. This guarantees him/her a maximum-security badge, the continuation of which only requires people to manage their social relationships in a safer way.

\subsection{Nowcasting}

Nowcasting, i.e., near real-time forecasting, refers to a combination of digital proximity tracing data from smartphones with additional data sources, e.g. Census data and Big Data, which allows authorities to forecast the risk that certain subjects/places, singly/by type, may likely become infected, in order to intervene in advance. This strategy matches the data collected by DPTT with the survey of territorial epidemiological information made through more extensive digital information retrieved from all kinds of sources (mobility data, search engines, social networks, credit card information, mobile network signals, telecom data, etc.), statistical, demographic data and information about places frequented (for work, leisure and school), data retrieved from hospitals, and swab-analysing labs and it may also rely on online anonymous surveys. Collected Big Data is elaborated with artificial intelligence algorithms and machine-learning risk assessment models in order to predict short-term spread and provide analyses for government's decisions. It is a massive calculation that makes it possible to predict with a certain probability whether a node of the social network = person (or territorial $=$ place), has been infected even if there are no symptoms, or if it could be expected soon because there is a high-risk profile (characteristics, behaviour, age, profession, etc.). The aim is to draw up an accurate and dynamic mapping of epidemic propagation at a neighbourhood-level that goes up to the single building for targeted testing. Implementing DPTT to support this strategy would also improve prediction accuracy and design appropriate protection measures for the most vulnerable individuals. Moreover, the possibility of revealing asymptomatic subjects allows the identification of potential new outbreak areas to anticipate or increase health efforts such as population screening. Finally, this may enable the authorities to concentrate on more urgent areas of intervention and to impose safeguards and red zones because an outbreak is expected, while easing measures in areas where an outbreak has been successfully avoided [35].

\section{Survey}

This section briefly describes the survey administered online in Italy in May 2020. The survey intended to understand what people think about the use of their smartphone for digital contact tracing, aimed at activating each DDEIS in the fight against COVID-19. It was designed with the specific purpose of collecting useful data to measure the degree of acceptance shown by the local population toward the use of DDEIS based on DPTT (as described in Section 3). Some of the data gathered from the survey were used to build the SEIR epidemiological model proposed in the following Section 5. Indeed, as shown by the literature review, several studies established models deriving from responses. These have been conceived to assess app acceptability [36], to investigate app adoption [37], to evaluate the incidence and evolution of COVID-19 [38-41], or to understand the impact on people's behaviour [42-45]. In the present work, the survey findings were used to compare possible impacts of different DDEIS on the diffusion dynamics of COVID-19 based on preferences for adoption scenarios.

\subsection{Survey Design}

The online survey consists of 22 questions. An introduction explains how data will be used, i.e., that data collection will be processed anonymously and exclusively for research purposes. Subsequently, four sections follow: (i) a description of DDEIS based on DPTT; (ii) questions to assess the degree of acceptance to DPTT; (iii) a targeted question to assess user's preference functions for each DDEIS, and (iv) demographic data for profile users.

Sections 1 and 3 aim to delineate preferred usage scenarios for each strategy, to tune them with a trade-off of associated details. Indeed, this allows users to identify details 
of implementation for each strategy, such as functionality; data processing; technology; why, where, and when to use DPTT; and with whom and what information to share, as well as possible adoption barriers. Afterwards they were asked to declare their willingness to opt into the DPTT on their device for the purpose of participating in each strategy. This implicitly assumes that the strategy in which the user agrees to participate is defined according to the conditions previously expressed by the user himself.

\subsection{Target Population and Sample Size}

At the time of conducting the survey, the sample was based on approximately 584 entries. A dedicated section on the user profile collects information on gender, age, job, and experience with COVID-19. The population was asked if they had ever had contact with positive subjects, if they had been swabbed, if they tested positive, if they had symptoms, or if they had health problems that could complicate the response to the infection. Of the sample interviewed, 95.3\% stated that they had not had contact with COVID-19-positive people. More than half of the remaining $4.7 \%$, who had had contact with positive subjects, had not been tested. Of swabbed individuals, $73 \%$ were negative. Of the interviewed sample, $94.7 \%$ stated that they had never had symptoms from COVID-19. $84 \%$ of the positive population stated that they had not been swabbed. Of the remaining swabbed participants, $40 \%$ were found to be infected. Of the people surveyed, $87.8 \%$ did not suffer from any previous disease. The results are summarized in Table 1.

Table 1. Summary of obtained results.

\begin{tabular}{ccccccccc}
\hline & \multicolumn{3}{c}{ Contact with Positive People } & \multicolumn{3}{c}{ COVID-19 Symptoms } \\
\cline { 2 - 10 } Sample & \multicolumn{2}{c}{$\mathrm{N}^{\circ}$ Interviewed } & \multicolumn{2}{c}{$\%$} & \multicolumn{2}{c}{$\mathrm{N}^{\circ}$ Interviewed } & $\%$ \\
\cline { 2 - 10 } Size: 584 & Yes & No & Yes & No & Yes & No & Yes & No \\
\cline { 2 - 10 } & 28 & 556 & 4.7 & 95.3 & 31 & 553 & 5.3 & 94.7 \\
\hline tested & 11 & 17 & 39 & 61 & 5 & 26 & 16 & 84 \\
\hline infected & 3 & 8 & 27 & 73 & 3 & 2 & 60 & 40 \\
\hline
\end{tabular}

It should be mentioned that the statistical sample is not sufficiently representative of the entire national population. However, this has little effect on the validity of this work, since in this phase of the research, an approximate range for DDEIS adoption was needed and not an exact percentage of results, in order to assess the extent of the range for the model and thus obtain representative outputs.

\subsection{Key Questions in Feeding Data into the Model}

Only two key questions of the survey were considered in the present paper in order to feed data into the epidemiologic model. The purpose of the questions was to understand (i) the most important right to be guaranteed for users for each strategy and (ii) what kind of strategy they were willing to use. For details, see Appendices A and B.

With regard to question (i), for each of the DDEIS, citizens indicated their preferences and were allowed to choose simultaneously more than one option about certain rights such as privacy, health, work and study, and mobility. Figure 1 shows the users' choices in relation to DDEIS.

The results show that the majority (45\%) recognized health as the most important right to be guaranteed. This preference can be considered as almost evenly distributed for all DDEIS. The right to privacy follows, with $33 \%$ consensus of the population. Specifically, a slight majority considers it to be important to ensure privacy, especially for Early Detection and Surveillance strategies. The right to work was recognised as important for $11 \%$ of people interviewed. In particular, the majority considered this an important right to be guaranteed in the case of Protection and Nowcasting strategies. The right to mobility, with 
a consensus of $11 \%$ of respondents, appears to be evenly distributed in relation to the four DDEIS.

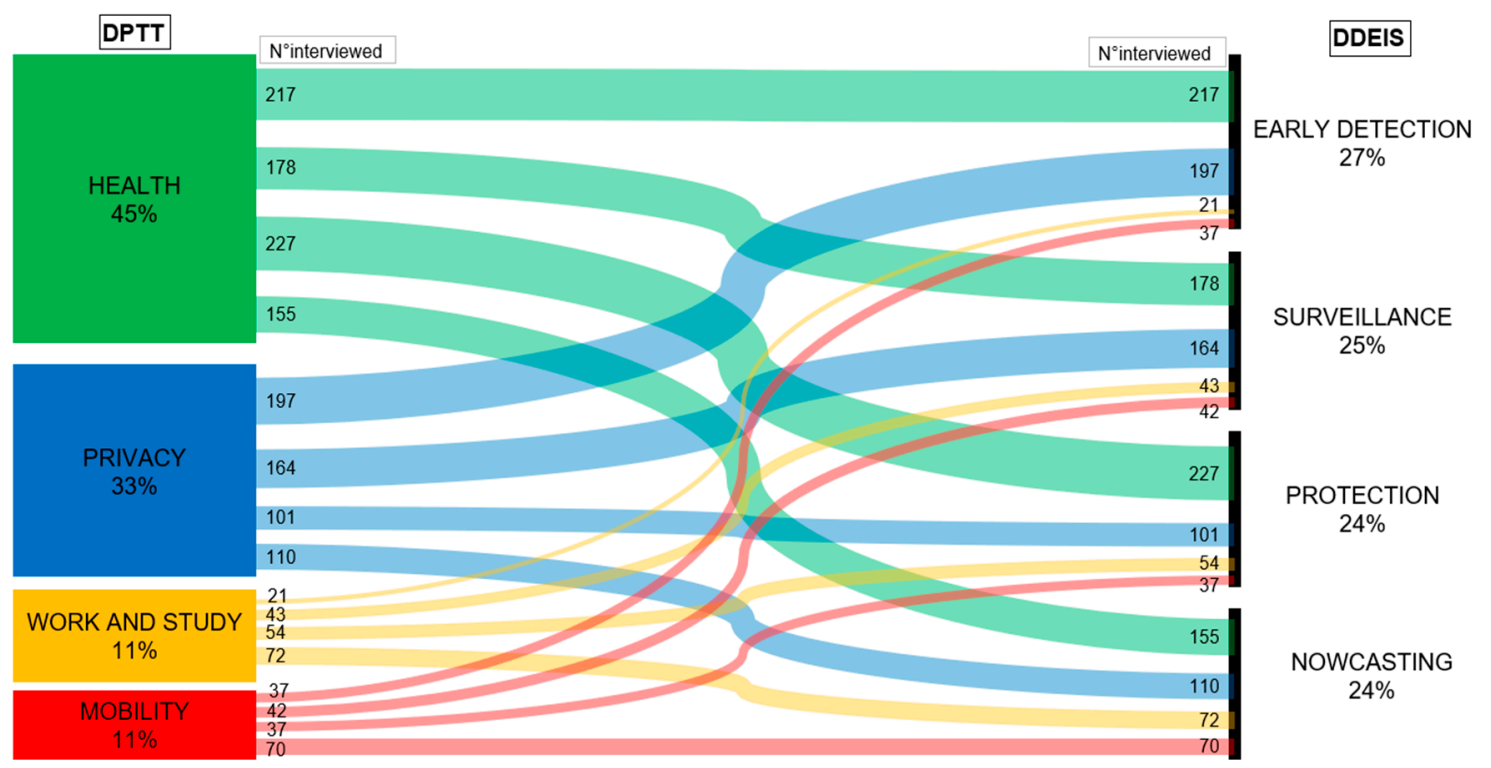

Figure 1. Users' preferences related to each strategy.

The outcomes of the survey (Figure 2) show that $86 \%$ of respondents would adopt the Early Detection strategy, while $72 \%$ of respondents would accept adopting the Surveillance strategy. As regards the Protection and Nowcasting strategies, the model (see Section 5 for further details) assumes, for the sake of simplicity, that the effect of these strategies on the epidemic dynamic can be modelled in the same way. Therefore, by averaging the percentage of acceptance of Protection and Nowcasting scenarios, it is notable that $87 \%$ of respondents would adopt these combined strategies.

YES

$86 \%$

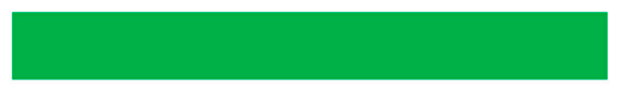

$72 \%$

$89 \%$

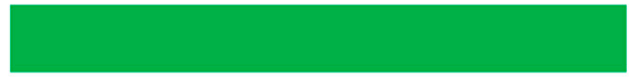

$84 \%$

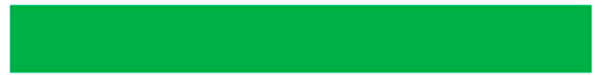

\section{EARLY} DETECTION

\section{SURVEILLANCE}

PROTECTION

NOWCASTING
NO

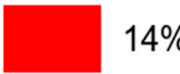

$28 \%$

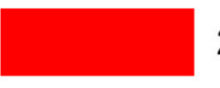

$11 \%$

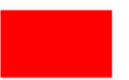

$16 \%$

Figure 2. Degree of acceptance of the proposed Data-Driven Epidemic Intelligence Strategies (DDEIS).

In a forthcoming paper, the full survey data will be analysed in greater detail, as this is currently under study to provide as wide a sample as possible. Moreover, the objective of this paper is not to analyse the results of the questionnaire, as this was used primarily as an initial support for the SEIR model of the DDEIS dynamic. Indeed, the survey allows us to estimate a set of parameters for the modelling of epidemic dynamics. Specifically, the percentages of adhesion to the different strategies inferred from the survey were adopted as input parameters for the model. Where a verification of the effectiveness of the DDEIS is necessary, the input parameters in the model should be modified with country-specific 
data to mirror the local context. Currently, the population sample is still limited; however, even if this is not extensive at the time of writing, it allows us to obtain plausible results to feed into the model.

\section{Epidemic Dynamic Model}

This section investigates the effects of the DDEIS described in Section 3 on the epidemic dynamics for reducing the spread of the infection.

As suggested by the WHO [46], the development of a mathematical model for the dynamic of the epidemic covers a key role in assessing and comparing the effectiveness of different public health intervention measures to control the disease and can be considered a necessary practice to provide valuable information for public health policymakers [47]. A large number of mathematical models [19,48-53] have been widely adopted to investigate the dynamics of COVID-19 disease. To this aim, a deterministic compartment model has been developed based on the well-known SEIR (Susceptible-Exposed-InfectiousRecovered) model [54-57]. The model developed in this work is an extended version of the SEIR model, a simplistic compartment model describing the flow of people through four mutually exclusive compartments. Our model assumes a population composed of individuals subdivided into the following mutually exclusive nine compartments:

- $\quad$ Susceptible (S): individuals able to contract the COVID-19 disease;

- $\quad$ Exposed (E): Susceptible individuals who have been exposed to infected people (asymptomatic, symptomatic, and exposed) and are able to infect susceptible individuals [21],

- $\quad$ Confined (C): optional compartment modelling the fraction of susceptible individuals removed from the dynamical model without moving to other compartments;

- Asymptomatic (A): Infected individuals showing mild or no symptoms;

- $\quad$ Symptomatic (I): Infected individuals showing symptoms related to the COVID-19 disease or any symptoms that might induce individuals to self-quarantine;

- Quarantined (Q): Infected and exposed individuals who self-isolate due to the application of DDEIS but still able to move to other compartments;

- Hospitalized (H): Infected individuals being treated in a hospital;

- $\quad$ Recovered (R): Individuals removed from the epidemic dynamics by recovering and not able to infect individuals;

- $\quad$ Deceased (D): Individuals who passed away due to the COVID-19 disease.

The model considers a closed, homogeneous, and well-mixed population of one hundred thousand individuals with no births and assumes the probability of reinfection of recovered individuals to be negligible, ensuring a lifelong immunity to the disease upon recovery. As a result, the model parameters that control the transitions of individuals across the compartments are assumed to be homogeneous over the modelled population. It is worth remarking that the model developed in this work is not aimed at accurately modelling the epidemic dynamics since most of the dynamical factors of the epidemic are inhomogeneous over the population and their values are still under debate in literature. However, this model can be considered a valuable tool to infer the effectiveness of DDEIS to control the spread of disease, thus making them effective for their purposes. The approach and the evolution equations describing the epidemic dynamic were developed to model the effects of the DDEIS introduced in this paper. It is therefore worth remarking that, although the approach is based on the well-known SEIR model, a novel set of dynamic equations were elaborated to mimic the flux of people among a set of compartments that represents the variety of scenarios according to the DDEIS proposed in this work. The novelty of the approach compared to previous works in literature lies in the definition of new transitions of individuals between compartments that are not commonly adopted in SEIR-like models. As an example, comparing the model developed in this paper with the model adopted in [50], it can be noticed that, due to the complexity of the DDEIS proposed here and the aim of the paper, our model considers a larger number of compartments into 
which the population is subdivided and, as a result, a new set of transitions of individuals between compartments.

The transitions of individuals between different compartments are modelled by nine ordinary differential equations, describing the evolution of the population in each stage over time:

$$
\begin{gathered}
\frac{\mathrm{d} S}{\mathrm{dt}}=-\beta \mathrm{S} \frac{\mathrm{A}+f \mathrm{I}+\mathrm{E}}{\mathrm{N}}-c \varepsilon S, \\
\frac{\mathrm{d} \mathrm{E}}{\mathrm{dt}}=c \varepsilon S, \\
\frac{\mathrm{dA}}{\mathrm{dt}}=(1-k-j)(1-h) \gamma \mathrm{E}-(1-k-j) \delta \mathrm{A}-(k+j) \varepsilon \mathrm{A}, \\
\frac{\mathrm{dI}}{\mathrm{dt}}=(1-k-j) h \gamma \mathrm{E}-(1-w-l)(1-n)(1-g) \mathrm{I} \eta-(w+l) \mathrm{I} \zeta \\
\frac{\mathrm{dQ}}{\mathrm{dt}}=(1-w-l)(1-n) g \delta \mathrm{I}-(1-w-l) n \varepsilon \mathrm{I}, \\
\left.\frac{\mathrm{d}}{w}+l\right) \mathrm{I} \zeta+(1-w-l) n \varepsilon \mathrm{I}+(k+j) \varepsilon \mathrm{E}+(k+j) \varepsilon \mathrm{A}-b \mathrm{Q} \delta-(1-b) \mathrm{Q} \eta, \\
\frac{\mathrm{dH}}{\mathrm{dt}}=(1-w-l)(1-n)(1-g) \mathrm{I} \eta+(1-b) \mathrm{Q} \eta-\theta \mathrm{H}, \\
\frac{\mathrm{dD}}{\mathrm{dt}}=m \theta \mathrm{H},
\end{gathered}
$$

where the upper-case Latin letters denote the state variables (S, C, E, A, I, Q, H, R, D) introduced above and $\mathrm{N}$ is the total number of individuals.

The Greek letters in Equations (1)-(9) denote the transition rates between different compartments and are positive numbers (Table 1). In detail, the rates are described as follows:

- $\quad \beta$ is the transmission rate, i.e., the average number of contacts between an infected (asymptomatic, symptomatic, and exposed) and a susceptible subject per unit of time multiplied by the probability of disease transmission through the contact. The model assumes that an exposed individual can transmit the virus at early stages as confirmed by epidemiological investigations [57]. The $\beta$ value covers a key role in the spread of the disease, since its value controls the flow of susceptible into the other compartments.

- $\quad f$ is a factor denoting the relative probability of disease transmission in a contact between a symptomatic and a susceptible individual with respect to the one related to the contact between an exposed/asymptomatic and a susceptible individual. As suggested by Ferguson [58], it has been assumed that symptomatic individuals are $50 \%$ more infectious than asymptomatic and exposed individuals, i.e., $f=1.5$;

- $\quad \gamma, \eta, \zeta, \delta$, and $\theta$ denote the rate at which individuals move between compartments. Their values are related to the incubation time $t_{\gamma}=\gamma^{-1}$, the average time to hospitalization $t_{\eta}=\eta^{-1}$, the average remission time $t_{\delta}=\delta^{-1}$, the average time $t_{\zeta}=\zeta^{-1}$ that the symptomatic take to self-isolate without any interventions of the policymakers and the application of DDEIS, and $t_{\theta}=\theta^{-1}$ is the average length of stay in hospital after which an individual can heal or die;

- $\quad \varepsilon$ denotes the rate at which individuals (asymptomatic, symptomatic, and exposed) decide to self-isolate due to the effects of DDEIS. The lower-case Latin letters in Equations (1)-(9) denote the fraction of people who move towards a different compartment, as explained below:

- $\quad c$ is the fraction of susceptible individuals who self-isolate due to the use of the Protection and Nowcasting DPTT functions; 
- $\quad h, w, g$, and $b$ respectively denote the fraction of exposed becoming symptomatic, the fraction of symptomatic going in quarantine and the fraction of not-quarantined symptomatic $(g)$, and the fraction of quarantined symptomatic $(b)$ who recover without being hospitalized;

- $\quad m$ is the fatality rate, which controls the flow of individuals out of the compartment of hospitalized;

- $\quad k$ and $n$ respectively denote the fraction of exposed/asymptomatic and symptomatic moving into quarantine compartment thanks to the use of the Early Detection strategy (Section 3.1);

- $\quad j$ and $l$ respectively indicate the additional fraction of exposed/asymptomatic and symptomatic moving to the quarantine compartment due to the application of the Surveillance strategy (Section 3.2).

Assuming that people's behaviour does not change and policymakers do not intervene to reduce the spread of the disease in any way $(c, k, n, j, l=0)$, it is possible to model the flow of individuals between compartments as follows. The principal flow of individuals occurs between susceptible (S) and exposed (E), which in turn are able to infect susceptible) through contacts with infective individuals (exposed $\mathrm{E}$, symptomatic I, and asymptomatic A) at a rate given by $\beta$. After the incubation time $t_{\gamma}$, a fraction $h$ of the exposed (E) become symptomatic (I) and a fraction $(1-h)$ become asymptomatic (A). Then, a fraction $w$ of the symptomatic are sufficiently symptomatic to self-isolate and flow toward the compartment of quarantined $(\mathrm{Q})$ at a rate $\zeta$. A fraction $g$ of the rest of the symptomatic (I), i.e., the fraction $(1-w) g$ of the total number of symptomatic, can exit their compartment because they recover $(\mathrm{R})$ from infection at a rate $\delta$, while the fraction $(1-w)(1-g)$ are hospitalized $(\mathrm{H})$ at a rate $\eta$. The asymptomatic (A) are removed at a rate $\delta$ due to remission $(\mathrm{R})$, while a fraction $b$ of home-isolated individuals $(\mathrm{Q})$ leave their compartment upon recovery $(\mathrm{R})$ at a rate $\delta$ and the fraction $(1-b)$ are hospitalized $(\mathrm{H})$ at a rate $\eta$.

Finally, a fraction $m$ of hospitalized individuals $(\mathrm{H})$ recover from infection $(\mathrm{R})$, while the fraction $(1-m)$ pass away (D) at a rate $\theta$. Hereafter, this scenario is referred to as the No-DDEIS scenario.

The transition rates of people between different compartments are regulated by several parameters related to the probability of disease transmission, the clinical progression of the disease, epidemiological status of the individuals, and the proposed intervention measures. In order to gain insights about the effectiveness of the four DDEIS described in Section 3, this paper considers three different scenarios, each of which is characterised by different transition flows between compartments and, in some cases, taking into account the additional "confined" compartment in the model. For the sake of simplicity, the model combines the effect of Protection and Nowcasting strategies in the same scenario.

The effects of the different DDEIS can be modelled as follows:

- Early Detection: a fraction $k$ of exposed (E) and asymptomatic (A) individuals and a fraction $n$ of not self-isolated symptomatic individuals (I), i.e., a fraction $(1-w) n$ of symptomatic, become quarantined and are removed from the epidemics (Section 3.1); - Surveillance: an additional fraction $j$ of exposed (E) and asymptomatic (A) individuals and an additional fraction $l$ of symptomatic (I) are quarantined. This functionality allows better control over whether infected individuals stay in quarantine (Section 3.2); - Protection and Nowcasting: a fraction $c$ of susceptible individuals (S) move to the confined compartment $(\mathrm{C})$ and, similarly to the quarantine compartment, are removed from the epidemics but do not move to other compartments (Sections 3.3 and 3.4).

In case all the DDEIS are simultaneously implemented, the fraction of people moving between compartments is modified accordingly, as shown in Equations (1)-(9). As an example, if the Early Detection strategy is applied, the model includes an additional flow of a fraction $k$ of those exposed and asymptomatic towards the compartment of quarantined, while a fraction $n$ not self-isolated symptomatic goes into quarantine at a rate $\varepsilon$. We assume that the exposed and asymptomatic behave differently with respect to symptomatic $(k \neq n)$. 
It is worth remarking that our approach does not model intervention like massquarantine and lockdown, because the use of DPTT is aimed at avoiding the adoption of non-specific strategies, which might irremediably lead to negative impacts on the economy [59].

A representative stock-flow diagram of the proposed model assuming that all scenarios occur (where $c, k, n, j, l \neq 0$ ) is shown in Figure 3. This diagram shows how individuals move through each compartment in the model. For integration of Equations (1)-(9), the standard real-valued variable-coefficient ordinary differential equation solver based on lsoda from ODEPACK [60] has been used. We adopt the following initial conditions (at $t=0$ ) for state variables: $S(0)=99800, E(0)=100, I(0)=60, A(0)=40$ while the rest of state variables are null.

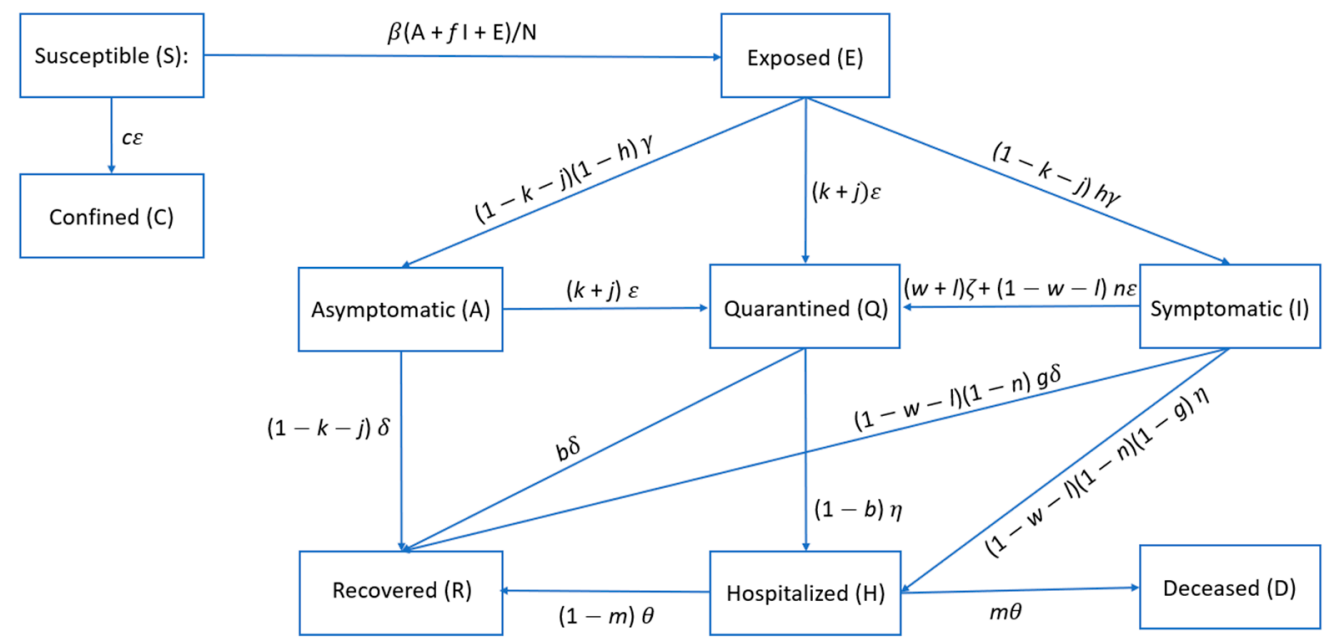

Figure 3. Schematic graphical diagram representing the flow of individuals between compartments.

The adopted model parameters are shown in Table 2, based on the estimates shown in Tang et al. and the results of the survey, as well as early reports and research on COVID-19 [58,61-66]. Moreover, our approach assumes that one-third of cases in the symptomatic compartments are sufficiently symptomatic to self-isolate $(\mathrm{w}=0.33)$ within 2 days of symptom onset and that $95 \%$ of quarantined individuals recover without being hospitalized. Furthermore, to improve the modelling, we assume that people start using the digital proximity tracing technology around seven days after the beginning of the epidemic using a smoothed step-like function. In detail, the values of the parameters $c, k, n$, $j$, and $l$ change as a logistic function swinging from the null value to the values listed in Table 2 at day 7 with a logistic growth timescale of one day. However, the model parameters have not been updated over time because we assume that no restrictions have been applied apart from those related to the use of the digital proximity tracing. It is worth remarking that, due to the limitation of compartment-based models and the increasing complexity in the nature of COVID-19 diseases and population models, validating the result of the model would be not practical in our case. As previously described, results can be interpreted as an indication of the power of DDEIS to control the disease. Finally, the model does not account for the limited capacity of the health care system to respond to the increasing number of hospitalized individuals. Specifically, the model does not limit the number of individuals within the hospitalized compartment to mimic the decreasing response to the health care system with epidemic evolution [67]. The model could mimic this effect by increasing the flux of individuals from the hospitalized to the deceased compartment by a factor related to the capacity of the health care system. However, since the health care system response relies upon several factors, such as hospital bed occupancy and the geographical distribution of hospitals, accounting for this effect would produce a loss of model generality. Since the approach developed in this paper is conceived to be as general 
as possible to fully figure out the effects of the proposed DDEIS, we therefore consider a constant response of the health care system during epidemic evolution.

Table 2. Model parameters.

\begin{tabular}{|c|c|}
\hline Parameter & Value \\
\hline$\beta$ & 0.75 \\
\hline$f$ & 1.5 \\
\hline$t_{\gamma}$ & 5.2 days \\
\hline$t_{\eta}$ & 3 days \\
\hline$t_{\zeta}$ & 2 days \\
\hline$t_{\delta}$ & 5.16 days \\
\hline$t_{\theta}$ & 12.5 days \\
\hline$h$ & 0.375 \\
\hline$w$ & 0.33 \\
\hline$g$ & 0.097 \\
\hline$b$ & 0.95 \\
\hline$m$ & 0.098 \\
\hline$k$ & 0.86 \\
\hline$n$ & 0.86 \\
\hline$j$ & 0.72 \\
\hline$l$ & 0.72 \\
\hline$c$ & 0.87 \\
\hline
\end{tabular}

The adopted value of $\beta$ (see Table 2) indicates that an infected individual can infect around 3.5 susceptible over the duration of infectiousness (that can be roughly estimated by the average of $t_{\gamma}, t_{\eta}$ and $t_{\delta}$ ). We run the simulation of the epidemic over a time period of 100 days.

\subsection{NO-DDEIS Scenario}

In the case where DDEIS are not adopted (where $c, k, n, j, l=0$ ), the confined compartment is not populated. Figure 4 shows the dynamical evolution of the number of individuals in each compartment of the epidemic model.

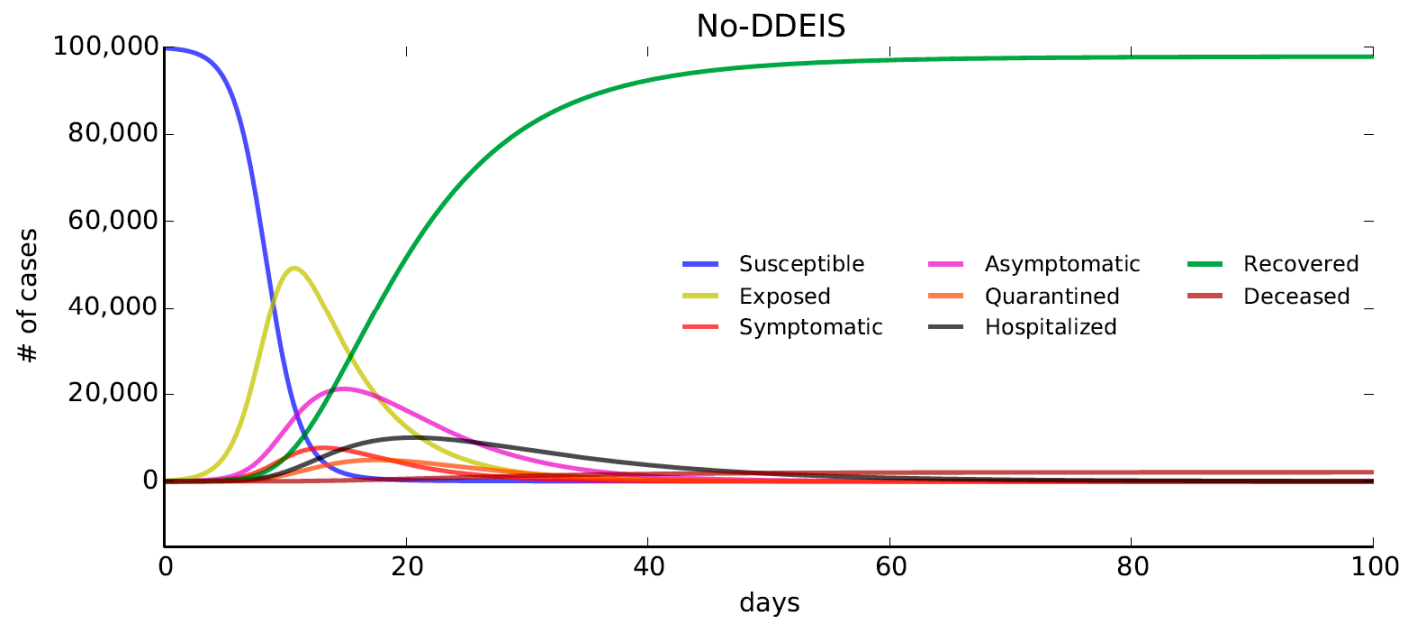

Figure 4. Evolution of the epidemic in the No-DDEIS scenario. 
The first days after the beginning of the epidemic are characterized by a remarkable increase of the exposed individuals due to the contacts between susceptible and infected. After nearly 15 days, the initial population of susceptible flow toward the compartment of exposed, asymptomatic, and symptomatic. The number of exposed, symptomatic, asymptomatic, quarantined, and hospitalized peaks at different times due to the different flow rates and decreases to null values after about 50 days. The fraction of deceased people with respect to the total number of individuals is $2.1 \%$ at the end of the simulation. Moreover, the evolution of the number of hospitalized peaks at a value of around $10 \%$ of the initial population of susceptible individuals.

\subsection{Early Detection}

The Early Detection scenario is modelled by taking into account flows of infected individuals (exposed, symptomatic, and asymptomatic) toward the compartments of quarantined. In detail, a fraction $k$ of individuals exposed (E) and asymptomatic (A) and a fraction $n$ of not-self-isolated symptomatic (I) are assumed to move towards the compartment of quarantine. In this case, we assume $c, j, l=0, k=0.86$, and $n=0.86$ (Table 2).

Figure 5 shows that nearly half of the population does not move from the compartment of susceptible due to the strong reduction of not-quarantined infected. The fraction of deceased people with respect to the total number of individuals is $0.5 \%$ at the end of the simulation. Comparing Figure 3 with Figure 2, it is possible to note a remarkable reduction of exposed individuals and the increase of quarantined people due to the implementation of the Early Detection strategy.

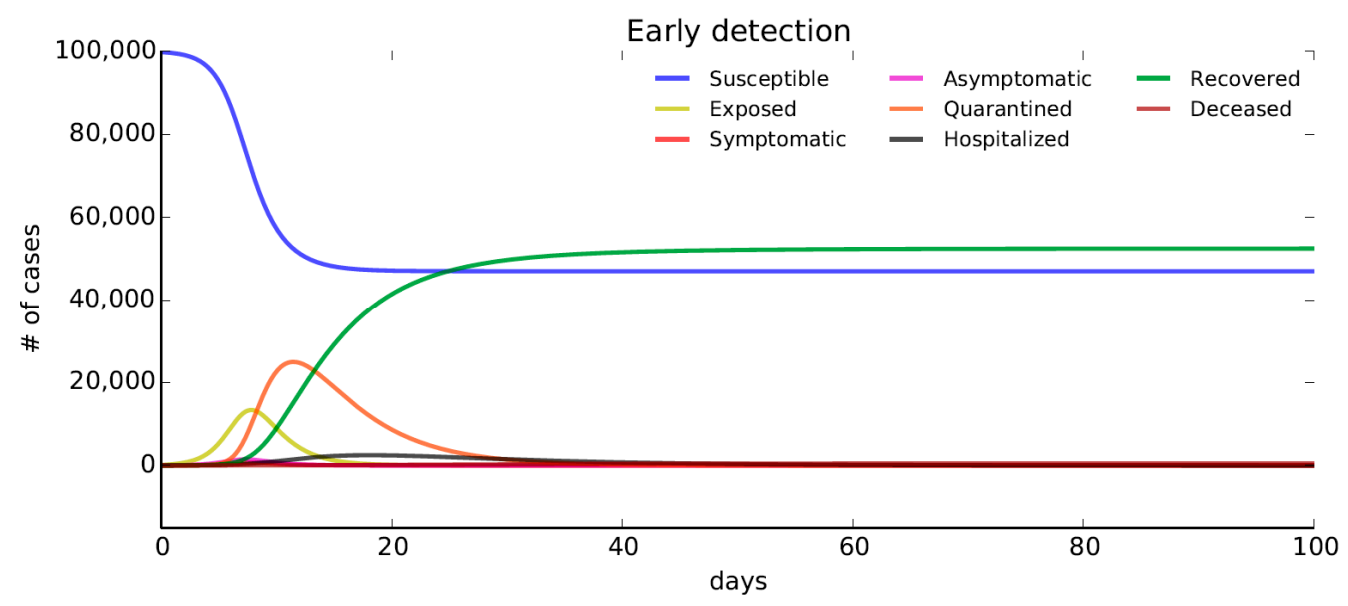

Figure 5. Evolution of the epidemic in the Early Detection scenario.

\subsection{Surveillance}

The Surveillance scenario is modelled by taking into account an additional flow of symptomatic individuals toward the compartments of quarantined. In this case, infected people are kept in quarantine thanks to the use of geofencing functions and police control.

In detail, we assume that a fraction $j$ of individuals exposed (E) and an additional fraction $l$ of symptomatic (I) move to the quarantine compartment. In this case, it has been assumed that $c, k, n=0, j=0.72$, and $l=0.72$ (Table 2).

Figure 6 shows that the flow of people out of the compartment of susceptible is similar to the one shown in Figure 2. In this case, more people are kept quarantined compared to the No-DDEIS scenario (Section 5.1). The fraction of deceased people with respect to the total number of individuals is $0.92 \%$ at the end of the simulation. The increase of the number of deceased people with respect to the Early Detection scenario implies that the Surveillance strategy is not as effective as the Early Detection one to reduce the spread of the diffusion, confirming evidence from The Washington Post's simulation illustration [68]. 


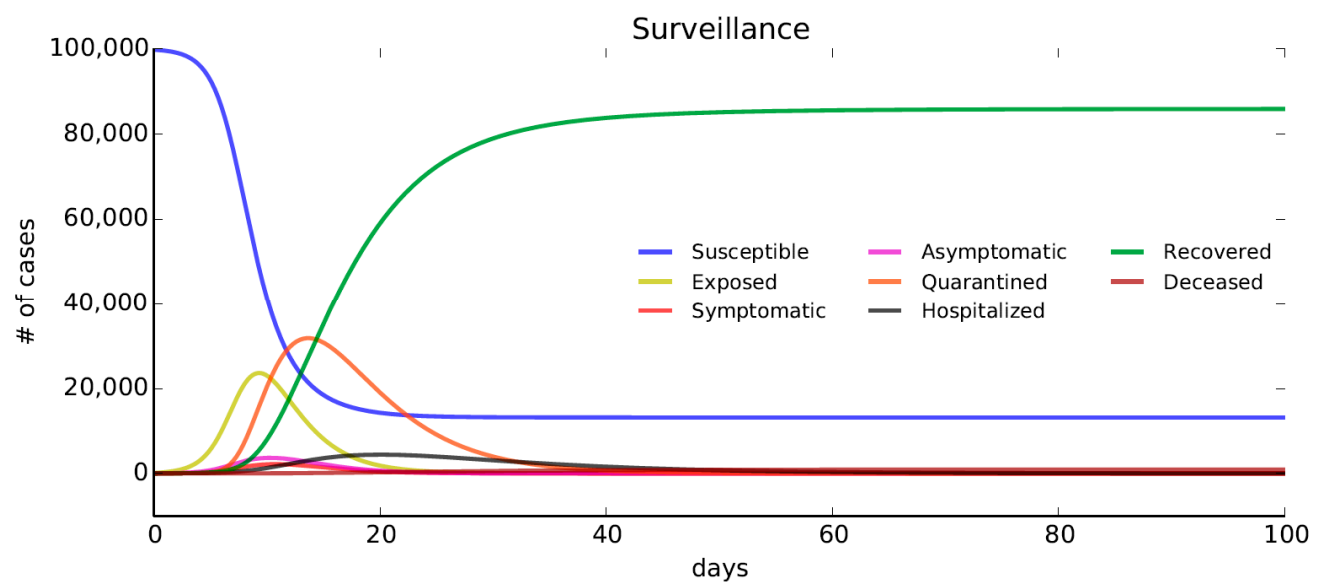

Figure 6. Evolution of the epidemic in the Surveillance scenario.

\subsection{Nowcasting and Protection}

The Nowcasting and Protection scenario is modelled as an additional flow of susceptible individuals toward the compartments of confined. As a result, more people are kept out of the epidemic without becoming infected since in the early stage of the epidemics, when the major part of individuals are susceptible, a fraction $c$ of susceptible self-isolate at a rate $\varepsilon$ (Equations (1) and (2)). In this case, we assume $k, n, j, l=0$ and $c=0.87$ (Table 2).

Figure 7 shows that the number of individuals in the compartment of confined is similar to the maximum number of quarantined people in Figures 3 and 4. However, in this case, confined people cannot become infected and stay in that compartment until the end of the epidemic, leading to a decrease in the number of infected and exposed people. The fraction of deceased people with respect to the total number of individuals is $0.58 \%$ at the end of the simulation, around two times the number of deceased individuals in the Early Detection scenario.

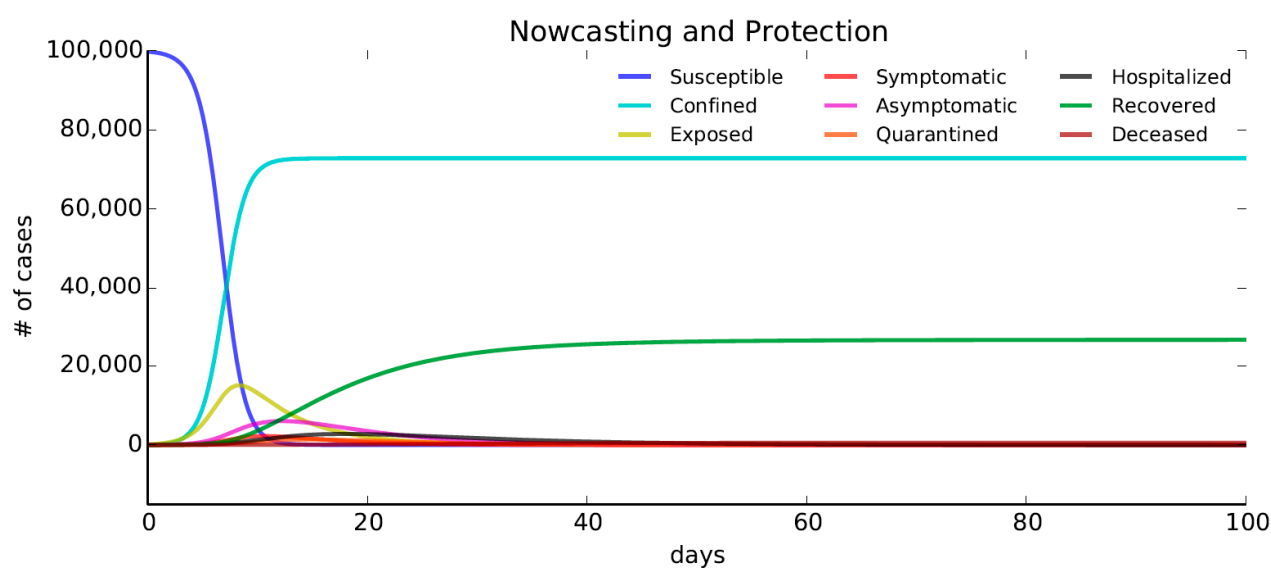

Figure 7. Evolution of the epidemic in the Nowcasting and Protection scenario.

\section{Results and Discussion}

This paper aims to clarify to what extent DDEIS could be effective and both technologically and socially suitable in reaching the objective of a swift return to normality for cities, guaranteeing public health safety and minimizing the risk of epidemic resurgence. The main contribution is the analysis of four different DDEIS from different perspectives, in terms of technical feasibility, spatial data requirements, social impact, personal data protection guarantees, and the power to mitigate infection. The key advantage is to infer the relative trend of the epidemic evolution among different scenarios in order to figure out the impact of the different DDEIS proposed and compare the effect of each strategy in terms of the reduction of the number of hospitalized and deceased individuals. By comparing 
the epidemic dynamics in each scenario, it is possible to note that the additional flux of people in the quarantine or confined compartments leads to a remarkable decrease in the number of infected individuals. These results confirm that the digital proximity tracing is a valuable intervention to reduce the spread of the disease without requiring any mass intervention such as lockdowns and avoiding overwhelming intensive care unit (ICU) capacity throughout the entire course of the epidemic. By targeting quarantine or confinement to those people potentially exposed or susceptible, i.e., users under Nowcasting and with active Protection strategy, the spread of disease is significantly reduced. Figure 8 shows the epidemic dynamical evolution if all the strategies are applied.

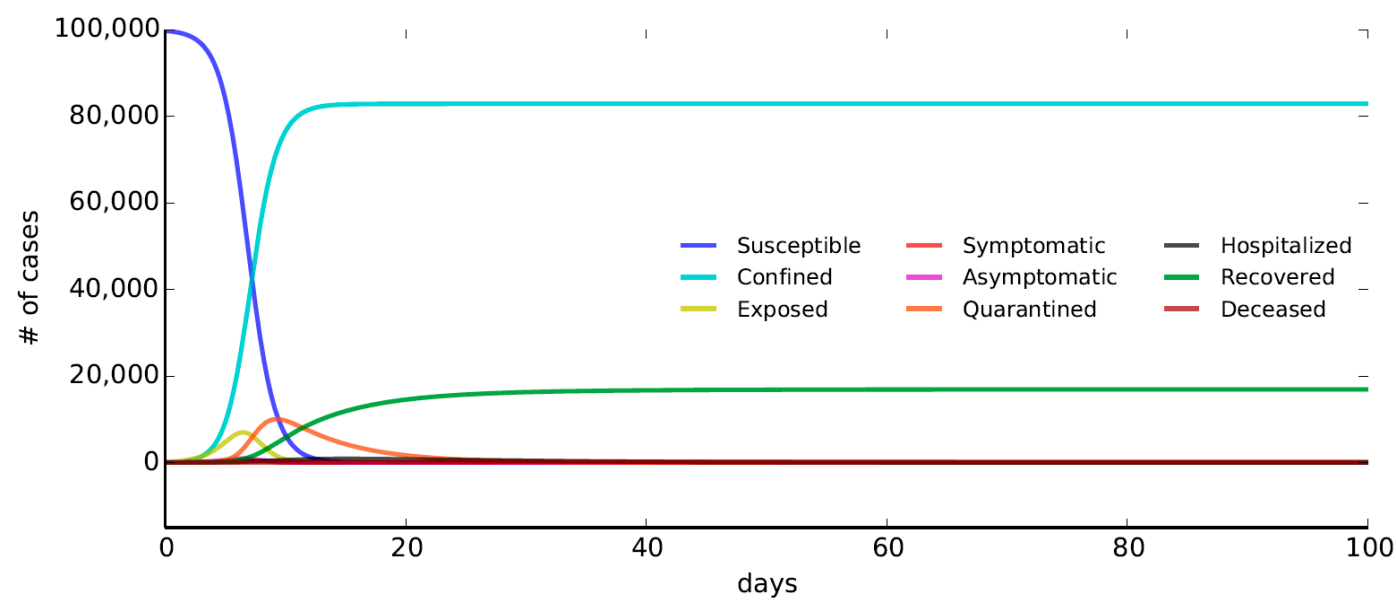

Figure 8. Evolution of the epidemic assuming that all the DDEIS strategies are adopted.

As expected, in this case, the number of deceased people reaches a value of $0.15 \%$ of the total number of individuals due to the strong reduction of not-confined and notquarantined infected.

By comparing the number of deceased individuals in each scenario considered in this analysis, Figure 9 shows that using all DDEIS can reduce the number of deceased to nearly $92 \%$ of the number of deceased in the No-DDEIS scenario.

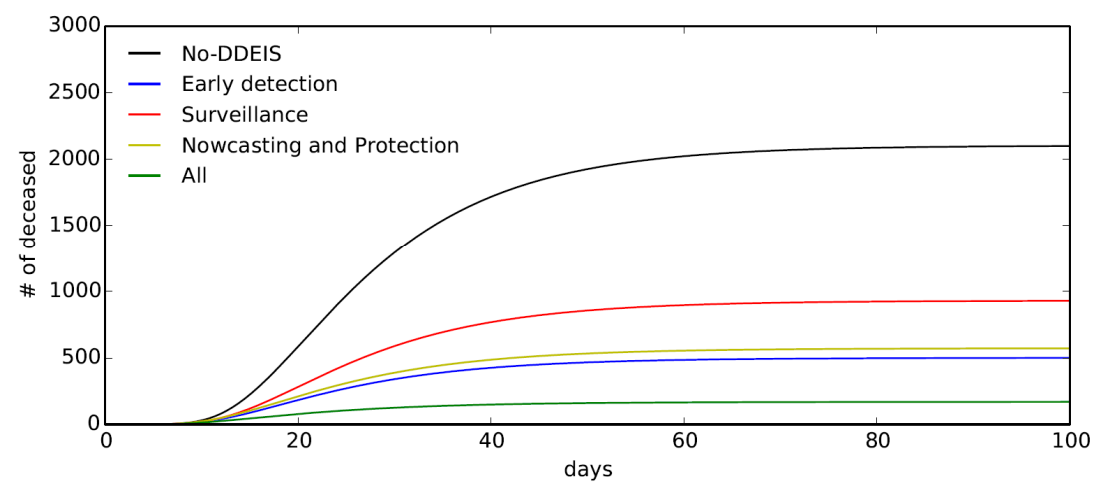

Figure 9. Number of deceased individuals in the considered scenarios along the epidemic evolution.

Figure 10 underlines the evolution of the number of hospitalized individuals resulting from the simulations. It can be noticed that the value peaks at nearly 20 days after the beginning of the epidemic. The use of the DDEIS leads to a remarkable reduction of the number of hospitalized individuals, strengthening the health system response to the epidemic and preventing hospital overcrowding. 


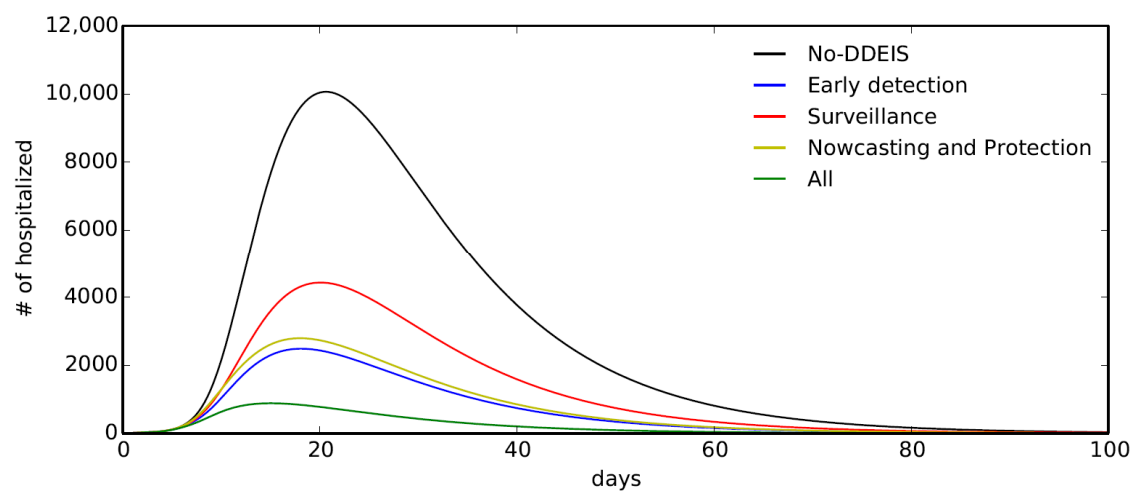

Figure 10. Number of hospitalized individuals in the considered scenarios along the epidemic evolution.

Assuming that the percentage of adhesion to the different strategies can likely be inferred from the survey, this means that any public authority interested in adopting the strategies can tailor the model to verify the impact of a particular DDEIS mix which most likely responds to the preferences of a specific cultural context and to the legal restrictions of interest. In this case, the comparison shows that the adoption of Early Detection and the combination of Nowcasting and Protection strategies lead to a higher reduction in hospitalized and deceased individuals with respect to the adoption of the Surveillance strategy, which indeed worldwide has always been applied in combination with others. This result is related to the differences in percentage of adhesion of the Early Detection or Nowcasting and Protection strategies (87\%, see Table 2$)$ compared to that of the Surveillance strategy (72\%, see Table 2). On the other hand, although Early Detection and Nowcasting and Protection seems to offer a comparable degree of impact, in order to function properly, the former implies a fully compliant and operating public health organizational infrastructure, whereas the latter is based on an automatic precaution mechanism distributed to each individual smartphone to prevent high-risk contacts and with the side-effect of relieving the operative management burden of traditional contact tracing.

\subsection{Limitations of the Model}

The greatest challenge in modelling the dynamic spread of specific diseases is to develop a model that fully captures the epidemiological progression of the epidemic by taking into account local demographic processes, the clinical and socioeconomic status of individuals, and the adopted intervention measures to mitigate the epidemic outbreaks. Due to the inherent complexity in including the effects of these aspects within the epidemic model, a trade-off between model complexity and accuracy should be achieved, bearing in mind the limitations of the model and how the results should be interpreted. The model developed in this paper is devoted to figuring out the relative impact of different DDEIS in the epidemic dynamic, thus carrying out a numerically controlled simulation to evaluate the relative fluxes of individuals among different compartments according to the different scenarios. This approach suffers from some uncertainties that might affect our results. The primary uncertainty of the model is related to the assumption of a homogeneous and well-mixed population, implying that all individuals in the population are assumed to have an equal probability of coming into contact with one another. This approach does not reflect human social structures, in which the majority of contact occurs within limited networks (i.e., the elderly are not adequately distanced from the younger members of their family and/or social circle). Additionally, the model simply assumes a closed population with no migration, births, or deaths from causes other than the epidemic and assumes immunity to the disease upon recovery. Furthermore, as previously described, the model does not limit the capacity of the health care system, thus implying that the transition between compartments is related to the epidemic properties (e.g., mortality, incubation time, etc.) and individuals' behaviour. Moreover, it still must be proved how DDEIS might work out at different stages of the contagion spread; in the earlier stage for containment 
before the breakout, subsequently for mitigation to delay the epidemic peak and avoid broader-scale social distancing, and lastly in the de-escalating transition phase to normality to avoid a resurgence of the epidemic. Furthermore, the accuracy of absolute numbers of individuals in each compartment computed by the model depends strongly on how accurate structural assumptions and the assumed parameters are. Therefore, care should be taken when interpreting the results of the epidemic dynamics in a single scenario due to the uncertainties in the parameter values and the limitation of the model. However, it is worth remarking that the main findings of the paper are related to the comparison of the impacts of the different DDEIS on the epidemic dynamic, thus providing insights on the efficiency of these strategies to mitigate epidemic effects.

\subsection{DDEIS Advantages and Drawbacks}

Eventually, analytical results demonstrate that DDEIS have the potential to make a substantial impact against the COVID-19 epidemic. However, it is worth underlining potentialities, drawbacks, and limits in implementing them, aiming toward an improved understanding of their applicability [69].

Firstly, there have been a number of debates and discussions about the risk of violation of personal privacy when adopting DPTT. There is no doubt that the possibility of a systematic control on a large scale of the position and the contacts of people can be considered a dangerous interference with private lives. Importantly, concerns about the data protection might be raised by the population. The European committee has identified principles and conditions that the DPTT must satisfy in order to avoid any violation of the privacy of each individual, regardless of the aim of technology use.

As regards the Early Detection strategy, if information about positives or potential positives were to be disseminated without strict anonymization, there would be a risk of causing social stigma, discrimination, or an unintentional witch-hunt. One solution to overcome such a risk is to conduct lottery-style randomized testing within a population [24]. Conversely, it must be considered if it should be left only up to the recipients of the warning message whether to choose to comply with it, as well as if users should be allowed to deactivate the tracking or to delete the data collected. This is because the time that potentially infected individuals take to contact the health authorities and isolate themselves and whether this is done before the onset of symptoms are crucial to control the spread. Indeed, this strategy might be deployed not only for those directly in contact with infected persons, but also along the chain of secondary and tertiary contacts. Although the positive infection status of the root individual should be confirmed by testing, it may not be required to implement preventative measures on potentially infectious derived contacts, since acting only after an official confirmation might be too slow to stop the spread. On the other hand, a system based on self-diagnosis is vulnerable to abuse since it affords the opportunity to broadcast panic. Therefore, the system may provide a different alert based on whether the infection report is self-made or uploaded by an approved testing service or public health professionals.

Early Detection adopts Bluetooth Low-Energy (BLE) signatures to obtain contact matching information, where signal strength between interacting devices is used to estimate distance. This works by collecting an anonymous list of constantly changing IDs of other devices that entered a certain proximity range from the infected one. These must run the same or a compatible app, which must also be active. Besides this, concerns regarding security vulnerabilities in Bluetooth technology have been posed [12]. Moreover, with BLE-based systems, no geolocation information is available compared to a strategy based on GPS, which can be integrated. Location tracking allows for the recording of path and positions of individuals to identify spatial interaction with others whose devices are also tracking their movements with GPS. This will allow for the retrieval of location history of an infected subject prior to the installation of the app to cross-check the recorded data up to the period of incubation. However, GPS technology alone does not guarantee the sensitivity of the reciprocal distance between devices offered by BLE, especially indoors. In 
addition, it could easily generate false positives, since the system may not discern whether the recorded movement was made on foot or by vehicle, creating the risk of warning people who had never been in real proximity to the virus. Finally, it is important to highlight that the Early Detection strategy makes smartphones a network of widespread sensors and that therefore its effective functioning would imply rapid follow-up testing (actuators) and professionals on hand to analyse samples. Furthermore, for this to be effective, the adoption of DPTT by a minimum number of users is required. This should reach at least $60 \%$ of the population [70], which is equivalent to $80 \%$ of people who own smartphones [9]. If the adoption of the technology does not exceed this threshold, it could lead to the unintentional consequence of lulling individuals into a false sense of security and thus lowering the attention given to other types of precautions. In this regard, if it is requested that users voluntarily join the tracking program, it may be necessary to provide incentives for its adoption.

Given all these complications and organizational, functional, and technological issues, the desired results from Early Detection implementation have been almost universally underwhelming with regard to initial hopes. Moreover, in the context of DDEIS, it is useful to note that the Early Detection strategy remains a public health measure, i.e., it does not offer the individual any means of prevention from coming into contact with infected people, which would only be known after contact has occurred.

With the Surveillance strategy, it is practical to include all the functions offered by Early Detection, which are overcome in favour of direct and constant control over the entire population through a centralized system. This strategy largely relies on the use of GPS integrated with Bluetooth, and the accuracy of location detection can be improved with position recordings by means of $Q R$ code scanning. In addition, this can be complemented by the monitoring and recording of infection-related symptoms at least once a day to support control decisions for epidemic management. Thus, it is simple to reach every individual present in a geographical area by broadcasting alerts or personalized information messages [71]. Obviously, for this strategy, there is no need to reach a minimum user number nor a voluntary adherence, as it is imposed on the entire population or on a targeted portion of it, e.g., arrivals from abroad; this is because it is automatically activated on devices or it is required by law. Beyond the vulnerability of the centralized servers to hacker attacks, the greatest limit of this strategy is the possibility of implemented control abuse. This may become a dangerous tool in the hands of authoritarian regimes that want to maintain power and create a constant state intrusion into individuals' lives at the expense of democracy, and in these cases, there is a real risk that this emergency solution lasts beyond the original crisis [13]. This risk is mitigated in countries, many Western, where digital surveillance is not accepted, since the privacy and freedom rights of individuals are preserved over government intrusion. In these countries, individuals have the control of their personal data. For example, they can ask about the erasure of data, rectification, and restriction of processing. For the same reason, only Early Detection strategies based on decentralized and voluntary contact-tracing apps have been implemented so far. In contrast, many Eastern countries have accepted some degree of privacy trade-off for the public good [24].

The Protection strategy could also incorporate the function offered by Early Detection if a decentralized data management solution is provided. Indeed, since the available data granularity reaches the range offered by BLE technology, this would also be valid for public places and indoor settings if these were also equipped with Bluetooth beacons or if they relied on cross-references with GPS data. For instance, it could alert the user to the possibility of having already crossed the path of a potentially infected person or a place where he has been, e.g., the same carriage on a train. However, it should be underlined that it primarily works as a preventative protective shield at the individual level to avoid the virus and not as a reminder of past contacts for people. Indeed, this informs individual decisions beforehand, which are directed according to the risk computed, which in turn should incorporate machine learning mechanisms to improve its accuracy. This strategy 
gives everyone a tool for knowledge to conduct risk prevention behaviours commensurate with what they consider appropriate for their own safety and that of their loved ones. This empowers people by giving them back the right to decide and freedom to socialize, and for these reasons it might gain public success. To function at its best, such a strategy would need to reach widespread adoption, which could take place if a social pressure mechanism arises such that it would be a social phenomenon emerging from the sum of individuals' behaviours that would push the non-compliant percentage of the population to adopt it. Compared to the centralized Surveillance strategy, the Protection strategy harnesses the greater number of data recorded on devices without the risk that a malicious authority or company could abuse or misuse it [33]. In fact, by sharing peer-to-peer information on the scored risk of infection at the same time for everybody, privacy would be equally breached in favour of all. This also means that information about whom you have encountered and where you have been and when would not be stored or shared, thereby preserving everyone's privacy.

At a technological level, this strategy requires a risk-prediction model based on an accurate risk scoring algorithm calculating a reliable probability that contact resulted in transmission (contagion/contamination). Indeed, to quantify the risk of contagion transmission, it would not be sufficient to consider only the degree of proximity and the duration of space sharing. Other parameters to be accounted for by the calculation may include the type of interaction, i.e., the activity risk conducted by agents, medical information (e.g., susceptibility of the recipients), the use of personal protective equipment or hand hygiene, the stage of infection, the infectivity of the transmitter, and more [30]. The score risk should also be tuned to avoid missing transmissions due to short but recurring contacts between individuals, none of which exceed the alert threshold. The algorithm should also set itself automatically for indoor places to consider for the absence of fresh air or for artificial ventilation. Lastly, a safety threshold should be set in favour of the precautionary principle and following indications from the fatality rate, which increases with age and is higher in people with comorbidities; this should be coherently adapted for these users [72]. Given such a wide possibility to fine-tune the model with comprehensive data, this strategy could be strongly recommended or even mandatory in high-risk workplaces such as a healthcare environment, where other strategies are not sufficient [73].

Finally, the Nowcasting strategy is designed to exploit the power of Big Data significantly better than other strategies. For instance, it is viable to feed simulations of risk scenarios that show how the contagion will develop within an error range and whether it will become an epidemic or disappear [74]. An example of the use of infection spread simulation at a microscale for decision support is presented by Esposito [75]. Moreover, geographic-based informative platforms, e.g., the COVID-19 Map implemented at Johns Hopkins University, could be part of the Nowcasting strategy. However, these for the public at large are mainly aimed at displaying quantitative information about the spatial development of the pandemic at glance; thus, their usefulness is limited by the aggregate nature of the provided data, which cannot properly drive individual behaviours at a local scale [3].

\section{Conclusions}

Data collection and epidemiological analysis are essential parts of assessing the impacts of mitigation strategies within cities alongside clinical research. In contributing to epidemic control, DPTTs were adopted in a large number of countries and cities.

The paper is a study of data-driven strategies to minimize the impact of global pandemics without extreme lockdown measures. An epidemiological model was fed with parameters, informed by responses to an online survey on user preferences for smartphone tracing. This system of nonlinear differential equations model enables predictions of death, peak hospital occupancy, and other measures of utility to policymakers. The paper's added value is to shed light on the importance of adopting model-driven strategies based on 
digital technologies to reduce the spread of the disease in cities by taking into account citizens' adherence and to speed up governments' evidence-based decisions. Indeed, this paper defined functions and purposes of different DDEIS based on DPTT and discussed possible implications for society through different scenarios for potential uses. A modified SEIR epidemiological model was developed with preliminary data from an online survey to simulate their impact on COVID-19 spread dynamics and allow for an initial comparison. However, this represents a first step in discussing the potentialities of DDEIS implementation using a simulation model; further research in this direction is needed. Lastly, key advantages and limitations regarding DDEIS implementation and technological and privacy concerns have also been pointed out.

Almost certainly, integrating a combination of DDEIS at flexible ranges in a wider decision-making for public health programs for epidemic containment and mitigation measures development would (i) support governments in designing more effective and scalable policies (e.g., to avoid an epidemic peak that overwhelms health-care services, to allocate personnel and resources according to need, and to precisely measure the social and economic impact to keep the effects on the economy manageable) [15]; (ii) foster an active involvement of the population in controlling propagation, e.g., improving social distancing and increasing the use of personal protective equipment in high-risk locations; (iii) enable a timely and precise backtrack of the transmission chain and rapid identification of outbreaks to enhance contact tracing and to address testing and quickening isolation and quarantine, e.g., understanding if certain areas need to enforce stronger measures such as lockdown; (iv) allow researchers to gain time to learn more about COVID-19, e.g., understanding the virus transmission dynamic and the different manifestations of the illness, identifying effective cures, and manufacturing drugs and vaccines.

Furthermore, this paper argues that a balanced combination between DDEIS desired results and the requirements of the cultural context of application should be pursued. This should complement rather than replace conventional public health strategy efforts, e.g., population screening for infection. For instance, it has been recognized that manual contact tracing also continues, given the low penetration of the DPTT among certain groups in the population, e.g., the elderly [8]. It is worth also remarking that additional non-pharmaceutical interventions such as mass quarantine and lockdown can successfully control the spread of the disease in a non-immune population [76]. However, the use of DPTT solutions is aimed at avoiding the adoption of non-tailored strategies, which might irremediably lead to negative impacts on the economy and psycho-social aspects [59]. At the same time, as underlined by the preliminary online survey, the acceptance of DDEIS by individuals depends on whether the public perceives them as effective, accurate, and trustworthy, avoiding mass surveillance and strictly limited in time to the duration of the current crisis.

Finally, since the choice of each strategy lies in the hands of local governments, based on national laws, citizens' preferences, and many other variables, it is not possible to define once and for all the best performer among them. However, this paper presents a thorough analysis of their potential impacts, together with an extended comparison between numerous aspects, prerequisites, and effects, which must be taken into consideration and weighed by decision-makers. This is needed in order to evaluate a more mindful adoption of DDEIS or possibly to reach a mixed trade-off that is both effective against the spread and adaptable to each local condition and context. This is the most significant finding of the study, which makes it a valuable support guide for decision-makers. Further developments will be devoted to structuring a more formal decision support tool.

Author Contributions: The paper has been carried out by authors as a common research work, nonetheless, Sections 1 and 2 were written by D.E. and A.S.; Section 3 by D.E.; Section 4 and appendix by S.S. and D.E.; Section 5 by G.D. and D.E.; Section 6 by G.D., D.E. and S.P.; Section 7 by I.P. and D.E. All authors have read and agreed to the published version of the manuscript.

Funding: This research received no external funding. 
Institutional Review Board Statement: Not applicable.

Informed Consent Statement: Under EU Regulation 2016/679 (General Data Protection Regulation "G.D.P.R"), any data collected from the survey was strictly anonymous and the authors were not able to identify those who responded.

Acknowledgments: We wish to thank Fabio De Maria and Ilenia Abbattista for their useful comments and the help for the set-up of the questionnaire.

Conflicts of Interest: The authors declare no conflict of interest.

\section{Appendix A}

Sampling Strategy

The sampling frame was based on a non-probability method. This form of sampling method is adopted when it is not possible to determine the probability of respondents participating or when respondents can choose to participate in the survey. For this reason, in this study, the type and number of respondents were not established in advance. Specifically, among the types of non-probability sampling, snowball sampling was chosen. and the survey was distributed through social media. This is used when the desired sample characteristic is difficult to find or when it is difficult or prohibitively expensive to reach a sufficiently large number of respondents by other means, such as simple random sampling [77]. The survey was built and shared online using Google Forms, a free tool available online. The survey was developed between March and May 2020 and tested in two trial sessions starting in April 2020. The first involved experts in the field, the second involved non-experts. The former trial session provided suggestions concerning the formulation of contents to guarantee users' acceptability, while some small formal changes were suggested by the latter to improve comprehension and readability.

\section{Appendix B}

\section{Key Questions for SEIR Model}

The full survey reached 584 Italian citizens and was carried out in the spring of 2020. Of all the 22 questions in the survey, only two were considered in the present study since these were sufficient for the modelling scope. Specifically, the questions selected were:

Question 1: Please indicate the most important user rights you think should be guaranteed for each strategy;

Question 2: Now that you have expressed your preferences about Why, How, When you would use digital tracking technology and with Whom and What information you would share, indicate whether you would be willing to use it.

Each question offers multiple answer options, and each was accompanied by a description of the DDEIS, as reported in the following Table A1, in order to provide a clear understanding of the different scenarios eventually obtained.

Table A1. DDEIS descriptions provided in the survey.

\begin{tabular}{cc}
\hline Digital Contact Tracing Technology & Description \\
\hline Early Detection & $\begin{array}{c}\text { This is only used after an infected person has been identified to find other potentially } \\
\text { infected people as rapidly as possible, including asymptomatic subjects. These can be } \\
\text { reached by health officials or anonymously by a phone message from the IT system. }\end{array}$ \\
\hline Surveillance & $\begin{array}{c}\text { This allows authorities to check that each person in the population follows restrictions and } \\
\text { laws linked to the COVID-19 emergency. }\end{array}$ \\
\hline Protection & $\begin{array}{c}\text { This allows each person to better manage social distancing; it warns on each person's } \\
\text { smartphone of the risk associated with a visit to a particular place or contact with a particular } \\
\text { person to allow for a more appropriate behaviour. }\end{array}$ \\
\hline Nowcasting & $\begin{array}{c}\text { This term refers to a combination of data from smartphones with Census Data and Big Data, } \\
\text { to allow authorities to forecast the risk that certain subjects/places, singly/by type, may } \\
\text { likely become infected in order to intervene in advance. }\end{array}$ \\
\hline
\end{tabular}




\section{References}

1. World Health Organization (WHO). Board, Global Preparedness Monitoring. A World at Risk: Annual Report on Global Preparedness for Health Emergencies; World Health Organization: Geneva, Switzerland, 2019.

2. World Health Organization (WHO). Infection Prevention and Control of Epidemic-and Pandemic-Prone Acute Respiratory Infections in Health Care; World Health Organization: Geneva, Switzerland, 2014.

3. COVID-19 Map-Johns Hopkins Coronavirus Resource Center. Available online: https://coronavirus.jhu.edu/map.html (accessed on 19 June 2020).

4. Nicola, M.; Alsafi, Z.; Sohrabi, C.; Kerwan, A.; Al-Jabir, A.; Iosifidis, C.; Agha, M.; Agha, R. The socio-economic implications of the coronavirus pandemic (COVID-19): A review. Int. J. Surg. 2020, 78, 185-193. [CrossRef]

5. International Monetary Fund. World Economic Outlook: The Great Lockdown; International Monetary Fund: Washington, DC, USA, 2020.

6. Flaxman, S.; Mishra, S.; Gandy, A.; Unwin, H.; Mellan, T.A.; Coupland, H.; Whittaker, C.; Zhu, H.; Berah, T.; Eaton, J.W.; et al. Estimating the effects of non-pharmaceutical interventions on COVID-19 in Europe. Nature 2020, 584, 257-261. [CrossRef]

7. Bonsall, D.; Fraser, C. Sustainable containment of COVID-19 using smartphones in China: Scientific and ethical underpinnings for implementation of similar approaches in other settings. GitHub, 16 March 2020.

8. European Commission. Mobile Applications to Support Contact Tracing in the EU's Fight Against COVID-19: Common EU Toolbox for Member States; European Commission: Brussels, Belgium, 2020.

9. Hinch, R. Effective Configurations of a Digital Contact Tracing App: A Report to NHSX. Available online: https:/ / github.com/ BDI-pathogens/covid-19_instant_tracing/blob/master/Report (accessed on 5 January 2020).

10. Jinjarak, Y.; Ahmed, R.; Nair-Desai, S.; Xin, W.; Aizenman, J. Accounting for Global COVID-19 Diffusion Patterns, January-April 2020 (No. w27185); National Bureau of Economic Research: Cambridge, MA, USA, 2020.

11. How South Korea Flattened the Coronavirus Curve. The New York Times. Available online: https://www.nytimes.com/2020/03/ 23/world/asia/coronavirus-south-korea-flatten-curve.html (accessed on 17 July 2020).

12. ACM Europe TPC. Statement on essential principles, practices for COVID-19 contact tracing applications. ACM TechNew, 8 May 2020.

13. Ada Lovelace Institute. Exit Through the App Store; Ada Lovelace Institute: London, UK, 2020.

14. Gasser, U.; Ienca, M.; Scheibner, J.; Sleigh, J.; Vayena, E. Digital tools against COVID-19: Taxonomy, ethical challenges, and navigation aid. Lancet Digit. Health 2020, 2, E425-E434. [CrossRef]

15. Anderson, R.M.; Heesterbeek, H.; Klinkenberg, D.; Hollingsworth, T.D. How will country-based mitigation measures influence the course of the COVID-19 epidemic? Lancet 2020, 395, 931-934. [CrossRef]

16. World Health Organization (WHO). Contact Tracing in the Context of COVID-19; World Health Organization: Geneva, Switzerland, 2019; pp. 1-7.

17. European Center for Disease, Prevention and Control. Coronavirus Disease 2019 (COVID-19) in the EU/EEA and the UK-Eighth Update; European Center for Disease, Prevention and Control: Solna, Sweden, 2020.

18. Gandhi, M.; Yokoe, D.S.; Havlir, D.V. Asymptomatic transmission, the Achilles' heel of current strategies to control Covid-19. N. Engl. J. Med. 2020, 382, 2158-2160. [CrossRef]

19. Hellewell, J.; Abbott, S.; Gimma, A.; Bosse, N.I.; Jarvis, C.I.; Russell, T.W.; Munday, J.D.; Kucharski, A.J.; Edmunds, W.J.; Sun, F.; et al. Feasibility of controlling COVID-19 outbreaks by isolation of cases and contacts. Lancet Glob. Health 2020, 8, e488-e496. [CrossRef]

20. Jones, T.C.; Mühlemann, B.; Veith, T.; Biele, G.; Zuchowski, M.; Hoffmann, J.; Stein, A.; Edelmann, A.; Corman, V.M.; Drosten, C. An analysis of SARS-CoV-2 viral load by patient age. medRxiv 2020. [CrossRef]

21. Ganyani, T.; Kremer, C.; Chen, D.; Torneri, A.; Faes, C.; Wallinga, J.; Hens, N. Estimating the generation interval for COVID-19 based on symptom onset data. medRxiv 2020. [CrossRef]

22. He, X.; Lau, E.H.Y.; Wu, P.; Deng, X.; Wang, J.; Hao, X.; Lau, Y.C.; Wong, J.Y.; Guan, Y.; Tan, X.; et al. Temporal dynamics in viral shedding and transmissibility of COVID-19. Nat. Med. 2020, 26, 672-675. [CrossRef]

23. Tellis, G.J.; Sood, A.; Sood, N. Price of delay in Covid-19 lockdowns: Delays spike total cases, natural experiments reveal. SSRN Electron. J. 2020, 1-24. [CrossRef]

24. Ryan, M. In defence of digital contact-tracing: Human rights, South Korea and Covid-19. Int. J. Pervasive Comput. Commun. 2020, 16, 63. [CrossRef]

25. Oliver, N.; Lepri, B.; Sterly, H.; Lambiotte, R.; Deletaille, S.; De Nadai, M.; Colizza, V. Mobile phone data for informing public health actions across the COVID-19 pandemic life cycle. Sci. Adv. 2020, 6, eabc0764. [CrossRef] [PubMed]

26. Kissler, S.M.; Tedijanto, C.; Goldstein, E.; Grad, Y.H.; Lipsitch, M. Projecting the transmission dynamics of SARS-CoV-2 through the postpandemic period. Science 2020, 368, 860-868. [CrossRef]

27. In Coronavirus Fight, China Gives Citizens a Color Code, With Red Flags. The New York Times. Available online: https: / / www.nytimes.com/2020/03/01/business/china-coronavirus-surveillance.html (accessed on 6 June 2020).

28. China Suppressed Covid-19 with AI and Big Data. Asia Times. Available online: https://asiatimes.com/2020/03/ china-suppressed-covid-19-with-ai-and-big-data/?fbclid=IwAR2xy3r_0MZz-XYg_drbqZC4OxmLquZTciqLp9wSz49e-72 ehwnKVZhoHzI (accessed on 6 June 2020).

29. Taiwan Phone Tracking System Monitors 55,000 Under Coronavirus Quarantine-Quartz. Available online: https:/ /qz.com/18 25997 / taiwan-phone-tracking-system-monitors-55000-under-coronavirus-quarantine/ (accessed on 6 June 2020). 
30. Esposito, D.; Schaumann, D.; Camarda, D.; Kalay, Y.E. Multi-agent modelling and simulation of hospital acquired infection propagation dynamics by contact transmission in hospital wards. In Advances in Practical Applications of Agents, Multi-Agent Systems, and Trustworthiness: The PAAMS Collection; Demazeau, Y., Holvoet, T., Corchado, J.M., Costantini, S., Eds.; Springer: Cham, Switzerland, 2020.

31. Van Doremalen, N.; Bushmaker, T.; Morris, D.H.; Holbrook, M.G.; Gamble, A.; Williamson, B.N.; Tamin, A.; Harcourt, J.L.; Thornburg, N.J.; Gerber, S.I.; et al. Aerosol and surface stability of SARS-CoV-2 as compared with SARS-CoV-1. N. Engl. J. Med. 2020, 82, 1564-1567. [CrossRef]

32. Kampf, G.; Todt, D.; Pfaender, S.; Steinmann, E. Persistence of coronaviruses on inanimate surfaces and their inactivation with biocidal agents. J. Hosp. Infect. 2020, 104, 246-251. [CrossRef]

33. Peer-to-peer AI-tracing of COVID-19-Yoshua Bengio. Available online: https://yoshuabengio.org/2020/03/23/peer-to-peer-aitracing-of-covid-19/ (accessed on 6 June 2020).

34. The Risks-Know Them-Avoid Them. Available online: https://www.erinbromage.com/post/the-risks-know-them-avoidthem (accessed on 6 June 2020).

35. Rossman, H.; Keshet, A.; Shilo, S.; Gavrieli, A.; Bauman, T.; Cohen, O.; Shelly, E.; Balicer, R.; Geiger, B.; Dor, Y.; et al. A framework for identifying regional outbreak and spread of COVID-19 from one-minute population-wide surveys. Nat. Med. 2020, 26, 634-638. [CrossRef]

36. Altmann, S.; Milsom, L.; Zillessen, H.; Blasone, R.; Gerdon, F.; Bach, R.; Abeler, J. Acceptability of app-based contact tracing for COVID-19: Cross-country survey evidence. JMIR mHealth uHealth 2020, 8, e19857. [CrossRef]

37. Thomas, R.; Michaleff, Z.A.; Greenwood, H.; Abukmail, E.; Glasziou, P. Concerns and misconceptions about the Australian Government's COVID Safe App: Cross-sectional survey study. JMIR Public Health Surveill. 2020, 6, e23081. [CrossRef]

38. Oliver, N.; Barber, X.; Roomp, K. The Covid19 impact survey: Assessing the pulse of the COVID-19 Pandemic in Spain via 24 questions. JMIR mHealth uHealth 2020, 8, e19857. [CrossRef]

39. Ojo, O.; García-Agundez, A.; Girault, B.; Hernández, H.; Cabana, E.; García-García, A.; Arabshahi, P.; Baquero, C.; Casari, P.; Ferreira, E.J. Coronasurveys: Using surveys with indirect reporting to estimate the incidence and evolution of epidemics. arXiv. 2020. Available online: https:/ / arxiv.org/abs / 2005.12783 (accessed on 6 June 2020).

40. Geldsetzer, P. Use of rapid online surveys to assess people's perceptions during infectious disease outbreaks: A cross-sectional survey on COVID-19. J. Med. Internet Res. 2020, 22, e18790. [CrossRef] [PubMed]

41. Nelson, L.M.; Simard, J.F.; Oluyomi, A.; Nava, V.; Rosas, L.G.; Bondy, M.; Linos, E. US public concerns about the COVID-19 pandemic from results of a survey given via social media. JAMA Intern. Med. 2020, 80, 1020-1022. [CrossRef] [PubMed]

42. Atchison, C.J.; Bowman, L.; Vrinten, C.; Redd, R.; Pristera, P.; Eaton, J.W.; Ward, H. Perceptions and behavioural responses of the general public during the COVID-19 pandemic: A cross-sectional survey of UK Adults. medRxiv 2020. [CrossRef]

43. Varshney, M.; Parel, J.T.; Raizada, N.; Sarin, S.K. Initial psychological impact of COVID-19 and its correlates in Indian community: An online (FEEL-COVID) survey. PLoS ONE 2020, 15, e0233874. [CrossRef]

44. Ho, H.Y.; Chen, Y.L.; Yen, C.F. Different impacts of COVID-19-related information sources on public worry: An online survey through social media. Internet Interv. 2020. [CrossRef]

45. Zanin, G.M.; Gentile, E.; Parisi, A.; Spasiano, D. A preliminary evaluation of the public risk perception related to the COVID-19 health emergency in Italy. Int. J. Environ. Res. Public Health 2020, 17, 3024. [CrossRef]

46. Egger, M.; Johnson, L.; Althaus, C.; Schöni, A.; Salanti, G.; Low, N.; Norris, S.L. Developing WHO guidelines: Time to formally include evidence from mathematical modelling studies. F1000Research 2017, 6, 1584. [CrossRef]

47. Huang, C.-Y.; Sun, C.-T.; Hsieh, J.-L.; Lin, H. Simulating SARS: Small-world epidemiological modeling and public health policy assessments. J. Artif. Soc. Soc. Simul. 2004, 7, 4.

48. Zhao, H.; Man, S.; Wang, B.; Ning, Y. Epidemic size of novel coronavirus-infected pneumonia in the Epicenter Wuhan: Using data of five-countries evacuation action. medRxiv 2020. [CrossRef]

49. Tang, B.; Bragazzi, N.L.; Li, Q.; Tang, S.; Xiao, Y.; Wu, J. An updated estimation of the risk of transmission of the novel coronavirus (2019-nCov). Infect. Dis. Model. 2020, 5, 248-255. [CrossRef]

50. Tang, B.; Wang, X.; Li, Q.; Bragazzi, N.L.; Tang, S.; Xiao, Y.; Wu, J. Estimation of the transmission risk of the 2019-nCoV and its implication for public health interventions. J. Clin. Med. 2020, 9, 462. [CrossRef]

51. Venkateswaran, J.; Damani, O. Effectiveness of testing, tracing, social distancing and hygiene in tackling Covid-19 in India: A system dynamics model. arXiv. 2020. Available online: https://arxiv.org/abs/2004.08859 (accessed on 6 June 2020).

52. Lyra, W.; do Nascimento, J.D.; Belkhiria, J.; de Almeida, L.; Chrispim, P.P.; de Andrade, I. COVID-19 pandemics modeling with SEIR(+CAQH), social distancing, and age stratification. The effect of vertical confinement and release in Brazil. medRxiv 2020. [CrossRef]

53. Kucharski, A.J.; Russell, T.W.; Diamond, C.; Liu, Y.; Edmunds, J.; Funk, S.; Eggo, R.M.; Sun, F.; Jit, M.; Munday, J.D.; et al. Early dynamics of transmission and control of COVID-19: A mathematical modelling study. Lancet Infect. Dis. 2020, 20, 553-558. [CrossRef]

54. Kermack, W.O.; McKendrick, A.G. Contributions to the mathematical theory of epidemics-I. Bull. Math. Biol. 1991, 53, 33-55.

55. Kermack, W.O.; McKendrick, A.G. Contributions to the mathematical theory of epidemics-II. Problem Endem. Bull. Math. Biol. 1991, 53, 57-87. [CrossRef] 
56. Kermack, W.O.; McKendrick, A.G. Contributions to the mathematical theory of epidemics-III. Further studies of the problem of endemicity. Bull. Math. Biol. 1991, 53, 89-118. [CrossRef]

57. Rothe, C.; Schunk, M.; Sothmann, P.; Bretzel, G.; Froeschl, G.; Wallrauch, C.; Zimmer, T.; Thiel, V.; Janke, C.; Guggemos, W.; et al. Transmission of 2019-nCoV Infection from an asymptomatic contact in Germany. N. Engl. J. Med. 2020, 382, 970-971. [CrossRef]

58. Ferguson, N.M.; Laydon, D.; Nedjati-Gilani, G.; Imai, N.; Ainslie, K.; Baguelin, M.; Bhatia, S.; Boonyasiri, A.; Cucunubá, Z.; Cuomo-Dannenburg, G.; et al. Report 9: Impact of Non-Pharmaceutical Interventions (NPIs) to Reduce COVID19 Mortality and Healthcare Demand; Imperial College London: London, UK, 2020.

59. McKibbin, W.; Fernando, R. The global macroeconomic impacts of COVID-19: Seven scenarios. Brookings, 2 March 2020.

60. Allan, B.; Lefantzi, S.; Ray, J. ODEPACK++: Refactoring the LSODE Fortran library for use in the CCA high performance component software architecture. In Proceedings of the Ninth International Workshop on High-Level Parallel Programming Models and Supportive Environments, Santa Fe, NM, USA, 26 April 2004.

61. Li, Q.; Guan, X.; Wu, P.; Wang, X.; Zhou, L.; Tong, Y.; Ren, R.; Leung, K.S.M.; Lau, E.H.Y.; Wong, J.Y.; et al. Early transmission dynamics in Wuhan, China, of novel coronavirus-infected pneumonia. N. Engl. J. Med. 2020, 382, 1199-1207. [CrossRef]

62. Akhmetzhanov, A.R.; Mizumoto, K.; Jung, S.; Linton, N.M.; Omori, R.; Nishiura, H. Estimation of the actual incidence of coronavirus disease (COVID-19) in emergent hotspots: The example of Hokkaido, Japan during February-March 2020. medRxiv 2020. [CrossRef]

63. Rees, E.; Nightingale, E.; Jafari, Y.; Waterlow, N.; Clifford, S.; Jombert, T.; Procter, S.; Knight, G. COVID-19 length of hospital stay: A systematic review and data synthesis. BMC Med. 2020, 18, 1-22. [CrossRef]

64. Al-Tawfiq, J.A. Asymptomatic coronavirus infection: MERS-CoV and SARS-CoV-2 (COVID-19). Travel Med. Infect. Dis. 2020, 101608. [CrossRef] [PubMed]

65. Day, M. Covid-19: Identifying and isolating asymptomatic people helped eliminate virus in Italian village. BMJ 2020, 368 , m1165. [CrossRef] [PubMed]

66. Du, R.-H.; Liu, L.-M.; Yin, W.; Wang, W.; Guan, L.-L.; Yuan, M.-L.; Li, Y.-L.; Hu, Y.; Li, X.-Y.; Sun, H.; et al. Hospitalization and critical care of 109 decedents with COVID-19 pneumonia in Wuhan, China. Ann. Am. Thorac. Soc. 2020, 17, 839-846. [CrossRef] [PubMed]

67. Klein, M.; Cheng, C.; Lii, E.; Mao, K.; Mesbahi, H.; Zhu, T.; Hupert, N. COVID-19 models for hospital surge capacity planning: A systematic review. Disaster Med. Public Health Prep. 2020, 1-8. [CrossRef]

68. Why Outbreaks Like Coronavirus Spread Exponentially, and How to "Flatten the Curve"—Washington Post. Available online: https:/ / www.washingtonpost.com/graphics/2020/world/corona-simulator/ (accessed on 17 July 2020).

69. Contact-Tracing Apps Are Not a Solution to the COVID-19 Crisis. Available online: https://www.brookings.edu/techstream/ inaccurate-and-insecure-why-contact-tracing-apps-could-be-a-disaster/ (accessed on 6 June 2020).

70. Ferretti, L.; Wymant, C.; Kendall, M.; Zhao, L.; Nurtay, A.; Abeler-Dörner, L.; Parker, M.; Bonsall, D.; Fraser, C. Quantifying SARS-CoV-2 transmission suggests epidemic control with digital contact tracing. Science 2020, 368. [CrossRef]

71. Raskar, R.; Schunemann, I.; Barbar, R.; Vilcans, K.; Gray, J.; Vepakomma, P.; Kapa, S.; Nuzzo, A.; Gupta, R.; Berke, A.; et al. Apps gone rogue: Maintaining personal privacy in an epidemic. arXiv 2020, arXiv:2003.08567.

72. Davies, N.G.; Klepac, P.; Liu, Y.; Prem, K.; Jit, M.; Eggo, R.M. CMMID COVID-19 working group. Age-dependent effects in the transmission and control of COVID-19 epidemics. medRxiv 2020. [CrossRef]

73. Esposito, D.; Schaumann, D.; Camarda, D.; Kalay, Y.E. A multi-agent simulator for infection spread in a healthcare environment. In International Conference on Practical Applications of Agents and Multi-Agent Systems; Springer: Cham, Switzerland, 2020; pp. 408-411.

74. Caligiuri, M.; Lombardo, E.; Piccoli, D. Link City: Oltre Lo Shock del Coronavirus. Un Modello Predittivo per Fronteggiare le Pandemie di Oggi e Prevenire Quelle di Domani; Società Italiana di Intelligence: Cosenza, Italy, 2020.

75. Esposito, D.; Schaumann, D.; Camarda, D.; Kalay, Y.E. Decision support systems based on multi-agent simulation for spatial design and management of a built environment: The case study of hospitals. In Proceeding of the 20th International Conference on Computational Science and Its Applications ICCSA 2020; Gervasi, B., Murgante, S., Misra, C., Garau, I., Blecic, D., Taniar, B., Apduhan, A., Rocha, E., Tarantino, C., Torre, Y.K., Eds.; Springer: Cham, Switzerland, 2020.

76. Gatto, M.; Bertuzzo, E.; Mari, L.; Miccoli, S.; Carraro, L.; Casagrandi, R.; Rinaldo, A. Spread and dynamics of the COVID-19 epidemic in Italy: Effects of emergency containment measures. Proc. Natl. Acad. Sci. USA 2020, 117, 10484-10491. [CrossRef]

77. Fricker, R.D. Sampling methods for web and e-mail surveys. In The Sage Handbook of Online Research Methods; Fielding, N., Lee, R.M., Blank, G., Eds.; SAGE Publications: London, UK, 2008; pp. 195-216. 